\title{
Mutual Fund Performance and Seemingly Unrelated Assets
}

\author{
Ĺuboš Pástor
}

Robert F. Stambaugh

University of Pennsylvania

Follow this and additional works at: https://repository.upenn.edu/fnce_papers

Part of the Finance Commons, and the Finance and Financial Management Commons

\section{Recommended Citation}

Pástor, L., \& Stambaugh, R. F. (2002). Mutual Fund Performance and Seemingly Unrelated Assets. Journal of Financial Economics, 63 (3), 315-349. http://dx. doi.org/10.1016/S0304-405X(02)00064-8 


\title{
Mutual Fund Performance and Seemingly Unrelated Assets
}

\begin{abstract}
Estimates of standard performance measures can be improved by using returns on assets not used to define those measures. Alpha, the intercept in a regression of a fund's return on passive benchmark returns, can be estimated more precisely by using information in returns on nonbenchmark passive assets, whether or not one believes those assets are priced by the benchmarks. A fund's Sharpe ratio can be estimated more precisely by using returns on other assets as well as the fund. New estimates of these performance measures for a large universe of equity mutual funds exhibit substantial differences from the usual estimates.
\end{abstract}

\section{Disciplines}

Finance | Finance and Financial Management 


\title{
Mutual fund performance and seemingly unrelated assets ${ }^{\star}$
}

\author{
Luboš Pástor a,*, Robert F. Stambaugh b,c \\ ${ }^{a}$ Graduate School of Business, University of Chicago, Chicago, IL 60637, USA \\ b The Wharton School, University of Pennsylvania, Philadelphia, PA 19104, USA \\ ${ }^{\mathrm{c}}$ The National Bureau of Economic Research
}

April 2001

\begin{abstract}
Estimates of standard performance measures can be improved by using returns on assets not used to define those measures. Alpha, the intercept in a regression of a fund's return on passive benchmark returns, can be estimated more precisely by using information in returns on non-benchmark passive assets, whether or not one believes those assets are priced by the benchmarks. A fund's Sharpe ratio can be estimated more precisely by using returns on other assets as well as the fund. New estimates of these performance measures for a large universe of equity mutual funds exhibit substantial differences from the usual estimates.
\end{abstract}

JEL classifications: G11, G12, C11

Keywords: Performance evaluation; Mutual funds; Bayesian analysis

*We are grateful to several anonymous referees, Chris Blake, Eugene Fama, Wayne Ferson, Anthony Lynch, Andrew Metrick, Dean Paxson, Toby Moskowitz, and seminar participants at the Federal Reserve Bank of New York, London Business School, Northwestern University, Ohio State University, University of Chicago, University of Pennsylvania, University of Rochester, Vanderbilt University, the 2000 NBER Summer Institute, the 2000 Portuguese Finance Network Conference, and the 2001 AFA Meetings for helpful comments. Research support from the Center for Research in Security Prices and Dimensional Fund Advisors is gratefully acknowledged (Pástor).

*Correspondence address. Graduate School of Business, University of Chicago, 1101 E. 58th St., Chicago, IL 60637, USA. Tel.: 773-8344080; fax: 773-7020458.

E-mail address: lubos.pastor@gsb.uchicago.edu (Ľ. Pástor) 


\section{Introduction}

A mutual fund's historical performance is often summarized by an estimate of its alpha or its Sharpe ratio. Alpha is defined as the intercept in a regression of the fund's excess return on the excess return of one or more passive benchmarks, while the Sharpe ratio is the fund's expected excess return divided by the standard deviation of the fund's return. These measures are usually estimated with historical returns on the assets that define them. That is, alpha is estimated using excess returns on the fund and the benchmarks, and the Sharpe ratio is estimated using the excess returns on the fund. This study demonstrates that an estimate of either performance measure can typically be made more precise by using historical returns on "seemingly unrelated" assets not used in the definition of that measure.

Alpha, for example, is usually estimated by applying ordinary-least-squares (OLS) to the regression

$$
r_{A, t}=\alpha_{A}+\beta_{A}^{\prime} r_{B, t}+\epsilon_{A, t},
$$

where $r_{A, t}$ is the fund's return in month $t, r_{B, t}$ is a $k \times 1$ vector containing the benchmark returns, and $\alpha_{A}$ denotes the fund's alpha. (Henceforth we use "returns" to denote rates of return in excess of a riskless interest rate or payoffs on zero-investment spread positions.) The choice of benchmarks is often guided by a pricing model, as in Jensen's (1969) pioneering use of the Capital Asset Pricing Model (CAPM) of Sharpe (1964) and Lintner (1965) to investigate mutual fund alphas relative to a single market-index benchmark. Other studies, beginning with Lehmann and Modest (1987), examine fund alphas with respect to a set of multiple benchmarks viewed as the relevant factors for pricing in a multifactor model, such as the Arbitrage Pricing Theory of Ross (1976).

As one special case, assume that the benchmark assets used to define alpha do indeed exactly price other passive assets. Consider the regression of a non-benchmark passive return $r_{n, t}$ on the benchmark returns,

$$
r_{n, t}=\alpha_{n}+\beta_{n}^{\prime} r_{B, t}+\epsilon_{n, t}
$$

where the correlation between $\epsilon_{A, t}$ and $\epsilon_{n, t}$ is positive. If the benchmarks price other passive assets, then $\alpha_{n}=0$. Now suppose that over the same sample period used to obtain the OLS estimate of $\alpha_{A}$, the OLS estimate of $\alpha_{n}$ is less than zero. Since the true value of $\alpha_{n}$ is zero, the negative estimate of $\alpha_{n}$ is fully attributed to sampling error. Given the positive correlation between $\epsilon_{A, t}$ and $\epsilon_{n, t}$, the OLS estimate of $\alpha_{A}$ is expected to contain negative sampling error as well, and this additional information can be used in estimating $\alpha_{A}$. 
As another special case, assume that the benchmarks used to define alpha have no pricing ability. To see how non-benchmark assets provide additional information about $\alpha_{A}$ in this case, consider a fund whose available return history is shorter than the histories of $r_{n, t}$ and $r_{B, t}$. The explosive growth of the mutual fund industry in recent years presents investors with many funds that have relatively short histories. Suppose that the OLS estimate of $\alpha_{n}$ computed for the sample period of the fund's available history is less than the OLS estimate of $\alpha_{n}$ computed for a longer sample period. Since the latter estimate is more precise, the first estimate is more likely to be less than the true (unknown) value of $\alpha_{n}$. Given the positive correlation between $\epsilon_{A, t}$ and $\epsilon_{n, t}$, the same can be said of the OLS estimate of $\alpha_{A}$ relative to its true value, and this information can be used in estimating $\alpha_{A}$. The additional information comes not through a pricing model, as in the previous case, but through the longer histories of the passive asset returns.

In the two special cases described above, $\alpha_{n}$ is assumed to be either zero or completely unknown. One may well prefer an intermediate version in which the benchmarks are believed to be relevant for pricing other passive assets, but not without error. In that general case, which we implement in a Bayesian framework, non-benchmark assets play a role that combines aspects of both previous cases. Additional information about $\alpha_{A}$ is provided by the extent to which the short-history estimate of $\alpha_{n}$ differs from zero as well as from its longhistory estimate. If the prior distribution for $\alpha_{n}$ is concentrated around zero, then most of the additional information is extracted from the difference of the short-history estimate from zero. As the prior spreads out, relatively more information is extracted from the difference between the short- and long-history estimates of $\alpha_{n}$.

Similar arguments apply when estimating a fund's Sharpe ratio. That is, a more precise estimate can be obtained using historical returns on more than just the fund. Perhaps the simplest setting in which returns on other assets can help estimate the mean and standard deviation of the fund return is that of Stambaugh (1997), who shows how assets with longer histories provide information about the moments of short-history assets. That principle enters the methods developed here, but so does the role of a pricing model. Consider the expected return on the fund, which can be written as

$$
E_{A}=\alpha_{A}+\beta_{A}^{\prime} E_{B},
$$

where $E_{A}$ and $E_{B}$ denote the means of $r_{A, t}$ and $r_{B, t}$. The sample mean of the fund's return, the usual estimate of $E_{A}$, can be obtained by replacing $\alpha_{A}$ and $\beta_{A}$ with their OLS estimates from (1) and by replacing $E_{B}$ with the sample mean of the benchmarks over the same sample period. To obtain a more precise estimate of $E_{A}$, we essentially use (3) and couple a longer- 
history estimate of $E_{B}$ with the more precise estimate of $\alpha_{A}$ discussed above. The estimate of $\alpha_{A}$, and hence the estimate of the fund's Sharpe ratio, relies on additional information provided by the return histories of the non-benchmark assets and incorporates beliefs about the degree to which those assets are priced by the benchmarks.

Our study does not recommend a particular set of benchmarks for defining alpha. Recent academic studies compute mutual fund alphas with respect to a single market benchmark (e.g., Malkiel (1995)) as well as sets of multiple benchmarks (e.g., Carhart (1997) and Elton, Gruber, and Blake (1996)). We compute alphas in both single-benchmark and multiplebenchmark settings. Alphas defined with respect to a single market benchmark may be of interest whether or not one believes in the CAPM. We offer just two of many examples of their use in practice: Morningstar, the leading provider of mutual fund information, reports alphas computed with respect to one of several broad market indexes; Capital Resource Advisors, one of the largest providers of performance information to institutional clients, reports alphas computed with respect to the S\&P 500 Index. Our approach allows one to estimate alpha under various assumptions about whether the benchmarks that define alpha price other passive investments. A common interpretation of alpha, one that implicitly places confidence in the benchmarks' pricing ability, is that it represents the skill of the fund's manager in selecting mispriced securities. A more general interpretation is that a positive alpha simply indicates that an investor can combine the fund and the benchmarks to obtain a Sharpe ratio higher than what can obtained by combining the benchmarks alone.

We investigate the performance of a large sample of equity mutual funds and find that the additional information about a fund's alpha and Sharpe ratio provided by seemingly unrelated assets can be substantial. Suppose, for example, that one has no confidence in the CAPM's pricing ability but nevertheless wishes to report a small-company growth fund's traditional alpha defined with respect to a single market benchmark. The absolute difference between the OLS estimate and an alternative estimate that incorporates information in nonbenchmark returns has a median value across such funds of $8.3 \%$ per annum. If instead one has complete confidence in the CAPM's pricing ability, then the median absolute difference in estimates is $7.2 \%$. In both cases, the alternative estimate is about three times more precise than the OLS estimate for the median small-company growth fund.

Across all funds in our sample, the median Sharpe ratio estimated the usual way, using the return history of just the fund, is 0.68 (annualized). When estimated using the additional information in seemingly unrelated passive assets, the median Sharpe ratio is no more than half that value. The new Sharpe-ratio estimates are typically four to five times more precise 
than the usual estimates. We also compare the rankings of funds based on the usual Sharperatio estimates to the rankings based on the new estimates. Of the funds with return histories of at least three years, only about $2 \%$ enter the top decile in both rankings. Of the funds that rank in the top decile based on the usual estimates, about $30 \%$ fall into the bottom two-thirds of the rankings based on the new estimates.

A number of studies observe that OLS estimates of mutual fund alphas are sensitive to the specification of the benchmarks that define those alphas. ${ }^{1}$ When the estimation of a fund's alpha incorporates non-benchmark assets, the definition of alpha typically becomes less important and, in some cases, even irrelevant. We estimate alphas defined with respect to the CAPM and with respect to the three Fama and French (1993) benchmark factors, which include size and value factors in addition to the market factor. When estimated using OLS, the median difference in alphas between the two models is $2.3 \%$ per annum for all funds and $8.1 \%$ for small-company growth funds. When the estimation incorporates nonbenchmark assets but does not rely on the benchmarks to price them, those values fall to $1.2 \%$ and $2.0 \%$.

If the benchmarks are assumed to price the non-benchmarks exactly, the estimates of a fund's alpha are identical under the CAPM and Fama-French models, even though the definitions of the alphas differ. This illustrates a general result. If alphas are defined with respect to different benchmarks but estimated using the same set of passive assets (benchmark and non-benchmark), then the estimates are identical if in each case the benchmarks are assumed to price the non-benchmark assets exactly. Loosely speaking, if you believe that some pricing model holds exactly and want a fund's alpha with respect to it, you need not identify the model. The appropriate estimate of alpha is then simply the estimated intercept in a regression of the fund's return on all of the passive assets. Such a regression can be likened to "style analysis," (e.g., Sharpe (1992)), in that the right-hand-side assets are included to capture multiple sources of variation (styles) in returns, regardless of whether only a subset of them might serve as the benchmarks in a pricing model. The intuition for the result is straightforward: adding to the right-hand side of the regression assets that are priced by others already included there lowers the residual standard deviation but leaves the true regression intercept unchanged.

As in numerous previous studies, we find that estimated alphas for the majority of equity mutual funds are negative. ${ }^{2}$ For each investment objective and each age group, we find a

\footnotetext{
${ }^{1}$ Examples include Lehmann and Modest (1987) and Grinblatt and Titman (1994). Roll (1978) provides a theoretical discussion of the potential sensitivity of alphas to benchmark specification.

${ }^{2}$ Grinblatt and Titman (1995) review the literature on mutual fund performance.
} 
posterior probability near $100 \%$ that the average of the funds' CAPM alphas is negative when the non-benchmark assets are excluded. Alphas for most funds remain negative when defined with respect to multiple benchmarks as well as when the information in the non-benchmark assets is used the estimation.

Section 2 discusses the econometric issues involved in obtaining our estimates of a fund's alpha and Sharpe ratio. Section 3 then reports results from computing alternative estimates of those measures for 2,609 equity mutual funds. Section 4 briefly reviews our conclusions.

\section{Estimating performance measures}

This section begins with some basic concepts underlying the use of seemingly unrelated assets to estimate a fund's performance measures. We then describe the details of our methodology, including our selection of the seemingly unrelated assets. Although the Bayesian framework we develop can accommodate informative prior beliefs about a fund's performance, all of the estimates we report in the next section are obtained using prior beliefs about a fund's performance that are "diffuse," or completely non-informative. That is, to be consistent with most of the academic literature as well as current practice, we allow the fund's track record to determine its estimated performance without any adjustment for prior beliefs about what one might think to be reasonable magnitudes for the performance measures. Such an approach is maintained here in order to focus on the contribution of seemingly unrelated assets. When considering fund performance in the context of fund selection or investment, an informative prior about performance is a sensible alternative. Baks, Metrick, and Wachter (2001) investigate the degree to which informative priors can preclude at least one actively managed fund from having a positive posterior mean for alpha and thereby looking attractive to an investor who can also invest in the passive benchmarks. Pástor and Stambaugh (2001) investigate the role of informative priors about fund performance, as well as pricing models, in selecting a portfolio of mutual funds.

\subsection{The role of seemingly unrelated assets}

Let $r_{N, t}$ denote the $m \times 1$ vector of returns in month $t$ on $m$ non-benchmark passive assets, so the multivariate version of the regression in (2) is written as

$$
r_{N, t}=\alpha_{N}+B_{N} r_{B, t}+\epsilon_{N, t}
$$


where the variance-covariance matrix of $\epsilon_{N, t}$ is denoted by $\Sigma$. Let $\sigma_{\epsilon}^{2}$ denote the variance of the disturbance $\epsilon_{A, t}$ in (1). Also define the regression of the fund's return on all $p(=m+k)$ passive assets,

$$
r_{A, t}=\delta_{A}+c_{A N}^{\prime} r_{N, t}+c_{A B}^{\prime} r_{B, t}+u_{A, t}
$$

where the variance of $u_{A, t}$ is denoted by $\sigma_{u}^{2}$. Substituting the right-hand side of (4) for $r_{N, t}$ in (5) gives

$$
r_{A, t}=\underbrace{\delta_{A}+c_{A N}^{\prime} \alpha_{N}}_{\alpha_{A}}+\underbrace{\left(c_{A N}^{\prime} B_{N}+c_{A B}^{\prime}\right)}_{\beta_{A}^{\prime}} r_{B, t}+\underbrace{c_{A N}^{\prime} \epsilon_{N, t}+u_{A, t}}_{\epsilon_{A, t}} .
$$

That is, using (1) and the fact that $r_{B, t}$ is uncorrelated with both $\epsilon_{N, t}$ and $u_{A, t}$ gives

$$
\alpha_{A}=\delta_{A}+c_{A N}^{\prime} \alpha_{N}
$$

and

$$
\beta_{A}=B_{N}^{\prime} c_{A N}+c_{A B}
$$

As explained below, the equality in (7) provides the key to understanding how additional information about $\alpha_{A}$ is provided by the $m$ non-benchmark assets, which are seemingly unrelated to $\alpha_{A}$ in that they are not required for its definition. Additional information about $E_{A}$ and $S_{A}$ is then provided, using (3), by the information about $\alpha_{A}$ as well as additional information about the expected returns of the $k$ benchmark assets, which are seemingly unrelated to $S_{A}$.

\subsubsection{Information about alpha: Intuition}

To see how additional information about $\alpha_{A}$ is provided by non-benchmark assets, consider initially a simplified setting in which the second-moment parameters $\beta_{A}, c_{A N}$, and $c_{A B}$ are viewed as known. Let $S$ denote the number of observations in the fund's return history, and define estimators of the intercepts in (1), (4), and (5) as

$$
\begin{aligned}
\bar{\alpha}_{A} & =(1 / S) \sum_{t=1}^{S}\left(r_{A, t}-\beta_{A}^{\prime} r_{B, t}\right), \\
\bar{\alpha}_{N} & =(1 / S) \sum_{t=1}^{S}\left(r_{N, t}-B_{N} r_{B, t}\right),
\end{aligned}
$$

and

$$
\bar{\delta}_{A}=(1 / S) \sum_{t=1}^{S}\left(r_{A, t}-c_{A N}^{\prime} r_{N, t}-c_{A B}^{\prime} r_{B, t}\right)
$$


Note using (8) that $\bar{\alpha}_{A}$ is also equal to the result from substituting $\bar{\delta}_{A}$ and $\bar{\alpha}_{N}$ into the right-hand side of $(7)$ :

$$
\begin{aligned}
\bar{\delta}_{A}+c_{A N}^{\prime} \bar{\alpha}_{N} & =(1 / S) \sum_{t=1}^{S}\left(r_{A, t}-\left(B_{N}^{\prime} c_{A N}+c_{A B}\right)^{\prime} r_{B, t}\right) \\
& =\bar{\alpha}_{A} .
\end{aligned}
$$

Suppose first that $\alpha_{N}$ is treated as a vector of unknown parameters, so that the benchmarks have no assumed pricing ability. Then $\alpha_{N}$ can be estimated more precisely than in (10) if the available history of $r_{N, t}$ and $r_{B, t}$ is longer than the $S$ observations in the fund's history. Substituting $\bar{\alpha}_{N}$ and $\bar{\delta}_{A}$ into the right-hand side of (7) gives $\bar{\alpha}_{A}$ as an estimator of

$\alpha_{A}$. Substituting the more precise estimator of $\alpha_{N}$ (along with $\bar{\delta}_{A}$ ) produces a more precise estimator of $\alpha_{A}$, since $\bar{\delta}_{A}$ is uncorrelated with either estimator of $\alpha_{N}$. (Note that, by construction, $u_{A, t}$ is uncorrelated with $\epsilon_{N, t}$.) Suppose instead that the benchmarks are assumed to price the non-benchmark assets exactly, so $\alpha_{N}=0$ and thus $\alpha_{A}=\delta_{A}$. Then both $\bar{\alpha}_{A}$ and $\bar{\delta}_{A}$ are unbiased estimators of $\alpha_{A}$, but the sampling variance of $\bar{\delta}_{A}, \sigma_{u}^{2} / S$, is less than or equal to the sampling variance of $\bar{\alpha}_{A}, \sigma_{\epsilon}^{2} / S$. In this case, the non-benchmark asset returns explain additional variance of the fund's return and thereby provide a more precise estimator of its alpha.

The basic idea is that a more precise estimator of $\alpha_{A}$ is obtained by evaluating the righthand side of (7) at $\bar{\delta}_{A}$ and a more precise estimator of $\alpha_{N}$ than $\bar{\alpha}_{N}$. A more precise estimator of $\alpha_{N}$ can be obtained by using a longer sample period, as in the case where the benchmarks are not assumed to have any pricing ability, or by simply setting $\alpha_{N}=0$, as in the case where the benchmarks are assumed to price the non-benchmark assets perfectly. When $\epsilon_{A, t}$ is correlated with the elements of $\epsilon_{N, t}$ (i.e. when $c_{A N} \neq 0$ ), then the difference between $\bar{\alpha}_{N}$ and a more precise estimator of $\alpha_{N}$ supplies information about the likely difference between $\bar{\alpha}_{A}$ and $\alpha_{A}$. When the more precise estimator of $\alpha_{N}$ relies on a longer history, the additional information about $\alpha_{A}$ is provided in essentially the same way that sample means of long-history assets provide information about expected returns on short-history assets, as in Stambaugh (1997).

\subsubsection{Estimating alpha: General methodological issues}

A Bayesian approach permits a range of prior beliefs about the ability of the $k$ benchmark assets to price the $m$ non-benchmark assets. A Bayesian setting is not required, however, to understand the basic issues governing the role of non-benchmark assets in estimating $\alpha_{A}$. 
We discuss here a number of those issues in the special cases where $\alpha_{N}$ is either restricted to be zero or left totally unrestricted.

Much of the intuition developed above when the slope coefficients $\beta_{A}, c_{A N}$, and $c_{A B}$ are known extends to the actual setting in which those parameters must be estimated. Equation (7) also holds when all quantities are replaced by OLS estimators based on the sample of $S$ observations. That is,

$$
\hat{\alpha}_{A}=\hat{\delta}_{A}+\hat{c}_{A N}^{\prime} \hat{\alpha}_{N}
$$

where $\hat{\alpha}_{A}, \hat{\alpha}_{N}$, and $\hat{\delta}_{A}$ are the OLS estimates of the intercepts in (1), (4), and (5), respectively, and $\hat{c}_{A N}$ is the OLS estimate in (5). As before, the information in non-benchmark assets is incorporated by replacing $\hat{\alpha}_{N}$ with a more precise estimator based either on a longer history or some degree of belief in a pricing model. When all parameters are unknown, substituting a more precise estimator of $\alpha_{N}$ can in some cases produce an estimator of $\alpha_{A}$ that is less precise than the usual estimator of the fund's alpha, $\hat{\alpha}_{A}$. For example, if one assumes that $\alpha_{N}=0$ and substitutes that value into (13) in place of $\hat{\alpha}_{N}$, the resulting alternative estimator of $\alpha_{A}$ is simply $\hat{\delta}_{A}$. The mean of $\hat{\delta}_{A}$ is $\alpha_{A}$, but the variance of $\hat{\delta}_{A}$ can exceed that of $\hat{\alpha}_{A}$. Since $c_{A N}$ must be estimated and $\hat{\delta}_{A}$ and the elements of $\hat{c}_{A N}$ are correlated, replacing $\hat{\alpha}_{N}$ with a lower-variance quantity need not lower the variance of $\hat{\alpha}_{A}$. Such an outcome is most likely to occur as the number of non-benchmark assets increases without a sufficient increase in the R-squared in (5). In essence, the degrees-of-freedom effect can outweigh the additional explanatory power. We use between five and seven non-benchmark assets, depending on the number of benchmarks, and we find that the information provided by those assets produces a more precise estimate of $\alpha_{A}$ for most funds in our sample. In the Bayesian framework explained below, we also apply a moderate degree of shrinkage to the slope coefficients in (5) to increase their precision and thereby enhance the information provided by the nonbenchmark assets. A potential direction for future research is the use of higher frequency data to increase the precision of the slope coefficients.

Suppose two researchers agree on an overall set of $p$ passive assets to include when estimating $\alpha_{A}$, but they disagree about the subset of those passive assets to designate as benchmarks for defining $\alpha_{A}$. Their chosen benchmark subsets might not even have any members in common. Moreover, suppose each researcher believes his benchmarks price the remaining passive assets perfectly. Then those researchers' estimates of $\alpha_{A}$ will be identical, even though their definitions of $\alpha_{A}$ are not. That is, the definition of $\alpha_{A}$ is irrelevant to its estimation if, for whatever benchmarks might be designated for defining $\alpha_{A}$, the remaining non-benchmark assets would be assumed to be priced exactly by those benchmarks. Perhaps ironically, if the benchmarks are not assumed to have perfect pricing ability, their designation 
becomes relevant not only for defining $\alpha_{A}$ but also for estimating it.

To understand the above statements, consider first the maximum-likelihood estimator (MLE) of $\alpha_{A}$ under the restriction that $\alpha_{N}=0$. If all regression disturbances are assumed to be normally distributed, independently and identically across $t$, then that estimator is given by $\hat{\delta}_{A}$, the OLS estimator of the intercept in (5), which does not depend on which of the $p$ assets are designated as the benchmarks. To see this, note that the disturbances $\epsilon_{N, t}$ and $u_{A, t}$ are uncorrelated and, given the normality assumption, independent. The likelihood function can therefore be expressed as a product of two factors, one for each regression. The restriction on $\alpha_{N}$ does not affect the MLE of $\delta_{A}$, which is $\hat{\delta}_{A}$, since $\alpha_{N}$ appears in the other factor. Substituting $\delta_{A}$ along with the restricted MLE of $\alpha_{N}$ (the zero vector) into the functional relation in (7) gives $\hat{\delta}_{A}$ as the MLE of $\alpha_{A}$ as well.

It can also be verified that $\hat{\delta}_{A}$ arises as the restricted estimator in a seemingly-unrelatedregression model, or SURM. ${ }^{3}$ That is, let regressions (1) and (4) jointly constitute a SURM, and consider the estimation of the model subject to the restriction $\alpha_{N}=0$. The restricted coefficient estimator requires the unknown joint covariance matrix of $\left(\epsilon_{A, t} \epsilon_{N, t}^{\prime}\right)$. If that matrix is replaced by the sample covariance matrix of the residuals from the first-pass unrestricted OLS estimation, the resulting "feasible" restricted SURM estimator of $\alpha_{A}$ is again simply $\hat{\delta}_{A}$. With no restriction on $\alpha_{N}$, then of course both the MLE and SURM estimator of $\alpha_{A}$ is simply the usual estimator $\hat{\alpha}_{A}$. When shrinkage is applied to the slope coefficients in (5), as in the Bayesian setting described below, the same type of result obtains. That is, the assumption $\alpha_{N}=0$ implies that the posterior mean of $\alpha_{A}$ is equal to the posterior mean of $\delta_{A}$, which doesn't depend on the designation of the benchmarks.

The principles governing the role of non-benchmark assets also apply when $\alpha_{A}$ is estimated by the generalized method of moments (GMM) of Hansen (1982). Let the parameter vector $\gamma$ contain the elements of $\delta_{A}, c_{A N}, c_{A B}, \alpha_{N}$, and $B_{N}$. The GMM estimator of $\gamma$ is obtained by minimizing $g(\gamma)^{\prime} W g(\gamma)$, where $g(\gamma)$ denotes the vector of $(1+m+k)+m(1+k)$ moment conditions,

$$
g(\gamma) \equiv\left(\begin{array}{c}
\frac{1}{S} \sum_{t \in F}\left(r_{A, t}-\delta_{A}-c_{A N}^{\prime} r_{N, t}-c_{A B}^{\prime} r_{B, t}\right)\left(\begin{array}{c}
1 \\
r_{N, t} \\
r_{B, t}
\end{array}\right) \\
\operatorname{vec}\left\{\frac{1}{T} \sum_{t=1}^{T}\left(r_{N, t}-\alpha_{N}-B_{N} r_{B, t}\right)\left(\begin{array}{c}
1 \\
r_{B, t}
\end{array}\right)^{\prime}\right\}
\end{array}\right),
$$

and $F$ denotes the subset of the periods $\{1, \ldots, T\}$ representing the fund's return history of

\footnotetext{
${ }^{3}$ Zellner (1962) introduces methods for estimating seemingly unrelated regressions. For a textbook treatment, including estimation under linear restrictions, see Theil (1971).
} 
length $S$. The first set of moment conditions in (14) corresponds to the regression in (5), and the second set corresponds to the regression in (4). The weighting matrix $W$ is block diagonal, since the disturbance in (4) is uncorrelated with that in (5). Consider the GMM estimates of the fund's alpha under the two cases discussed earlier. In the first case, with no restriction on $\alpha_{N}$, the above moment conditions exactly identify $\gamma$. Using the GMM estimate $\check{\gamma}$, the fund's estimated alpha is then $\check{\alpha}_{A}=\check{\delta}_{A}+\check{c}_{A N}^{\prime} \check{\alpha}_{N}$. Observe that $\check{\alpha}_{N}$ is based on the data for all $T$ periods, whereas $\check{\delta}_{A}$ and $\check{c}_{A N}$ are based on only the observations corresponding to the fund's history. In the second case, with the pricing restriction $\alpha_{N}=0$, the second set of moment conditions can be dropped and the fund's alpha is estimated simply as $\check{\alpha}_{A}=\check{\delta}_{A}$.

\subsubsection{Estimating the Sharpe ratio}

The $k$ benchmark assets as well as the $m$ non-benchmark assets are seemingly unrelated to a fund's Sharpe ratio, $S_{A}$, in that neither set of assets is related to the definition or usual estimate of $S_{A}$. The sample mean return on the fund obeys the relation,

$$
\bar{r}_{A}=\hat{\alpha}_{A}+\hat{\beta}_{A}^{\prime} \bar{r}_{B}
$$

where the OLS estimators $\hat{\alpha}_{A}$ and $\hat{\beta}_{A}$ and the sample mean vector of the benchmarks, $\bar{r}_{B}$, are computed using observations for the same $S$ periods used to estimate $\bar{r}_{A}$. A more precise estimator of $E_{A}$ can be obtained by replacing $\hat{\alpha}_{A}$ with the more precise estimator of $\alpha_{A}$ described above and by replacing $\bar{r}_{B}$ with the sample mean computed over a longer sample period. For example, when $\alpha_{N}$ is restricted to be zero, and hence $\alpha_{A}=\delta_{A}$, a simple alternative estimator of $E_{A}$ is $\hat{\delta}_{A}+\hat{\beta}_{A}^{\prime} \hat{E}_{B}$, where $\hat{E}_{B}$ is the sample mean vector of the benchmarks over a longer sample period of length $T$. In that case, additional information about $E_{A}$, and thus $S_{A}$, is provided both by the application of the pricing relation to the non-benchmark assets as well as by the longer history of the benchmark returns. This alternative estimator of $E_{A}$ differs somewhat from the Bayesian estimator we actually use, which uses shrinkage techniques to obtain a more precise estimator of $\beta_{A}$, but it illustrates simply the main sources of additional precision in estimating $E_{A}$. When no restriction is placed on $\alpha_{N}$, then the longer histories of both the benchmark and non-benchmark assets provide additional information about $E_{A}$ in the same manner as in Stambaugh (1997), with the additional feature that shrinkage techniques are again applied to slope coefficients.

The denominator of a fund's Sharpe ratio is $\sigma_{A}$, the standard deviation of the fund's return. The seemingly unrelated assets provide additional information about that parameter as well. As in Stambaugh (1997), the longer histories of those assets provide information 
about the volatility of the fund's return beyond what is provided by the fund's shorter return history. Although the Bayesian posterior mean of $S_{A}$ cannot quite be viewed as a ratio of separate estimates of $E_{A}$ and $\sigma_{A}$, the seemingly unrelated assets provide information about the latter parameters largely through the channels just described.

\subsection{The benchmark and non-benchmark assets}

Our set of benchmark and non-benchmark assets consists of eight portfolios constructed passively, in that their composition is determined using mechanical rules applied to simple, publicly available information. Monthly returns on these passive assets are constructed for the $35 \frac{1}{2}$-year period from July 1963 through December 1998. The sample period for any given fund, typically much shorter, is a subset of that overall period. We specify up to three benchmark series, consisting of the three factors constructed by Fama and French (1993), updated through December 1998. ${ }^{4}$ The first of these, MKT, is the excess return on a broad market index. The other two factors, SMB and HML, are payoffs on long-short spreads constructed by sorting stocks according to market capitalization and book-to-market ratio. We estimate "Fama-French" alphas, defined with respect to all three benchmarks, as well as "CAPM" alphas, defined with respect to just MKT.

When estimating CAPM alphas, SMB and HML become two of the non-benchmark series. Five additional non-benchmark series are used in the estimation of both CAPM and Fama-French alphas. The first of these, denoted as CMS, is the payoff on a characteristicmatched spread in which the long position contains stocks with low HML betas (in a multiple regression including MKT and SMB) and the short position contains stocks with high HML betas. The long and short positions are matched in terms of market capitalization and bookto-market ratio, and the overall spread position is formed from a set of triple-sorted equity portfolios constructed as in Pástor and Stambaugh (2000), who closely follow the procedures of Daniel and Titman (1997) and Davis, Fama, and French (2000). At the end of June of each year $s$, all NYSE, AMEX, and NASDAQ stocks in the intersection of the CRSP and Compustat files are sorted and assigned to three size categories and, independently, to three book-to-market categories. The nine groups formed by the intersection can be denoted by two letters, designating increasing values of size (S, M, B) and book-to-market (L, M, H). We then construct beta spreads within the four extreme groups of size and book-to-market: SL, $\mathrm{SH}, \mathrm{BL}$, and $\mathrm{BH}$. The stocks within each group are sorted by their HML betas and assigned

\footnotetext{
${ }^{4}$ We are grateful to Ken French for supplying these data.
} 
to one of three value-weighted portfolios. ${ }^{5}$ A spread within each group is constructed each month (from July of year $s$ through June of year $s+1$ ) by going long $\$ 1$ of the low-beta portfolio and short $\$ 1$ of the high-beta portfolio, and the value of CMS in month $t$ is the equally weighted average of the four spread payoffs in month $t$.

The second non-benchmark series, denoted as MOM, is the "momentum" factor constructed by Carhart (1997). At the end of each month $t-1$, all stocks in the CRSP file with return histories back to at least month $t-12$ are ranked by their cumulative returns over months $t-12$ through $t-2$. The value of MOM in month $t$ is the payoff on a spread consisting of a $\$ 1$ long position in an equally weighted portfolio of the top $30 \%$ of the stocks in that ranking and a corresponding $\$ 1$ short position in the bottom $30 \%$.

The remaining three non-benchmark assets, whose returns are denoted as IP1, IP2, and IP3, are portfolios constructed from a universe of 20 value-weighted industry portfolios formed using the same classification scheme as Moskowitz and Grinblatt (1999). The three portfolios mimic the first three principal components of the disturbances in multiple regressions of the 20 industry returns on the other passive returns: MKT, SMB, HML, CMS, and MOM. The vector of weights for IP1 is proportional to the eigenvector for the largest eigenvalue of the sample covariance matrix of the residuals in those regressions, and the other two portfolios are similarly formed using eigenvectors for the second and third eigenvalues.

The specification of non-benchmark assets must be somewhat arbitrary, but our selection of the five described above is based on several considerations. Recall that non-benchmark assets supply information about $\alpha_{A}$, the fund's alpha, when they explain additional variance of the fund's returns, i.e. when $c_{A N} \neq 0$. Also, except when the benchmarks are assumed to price the non-benchmark assets perfectly, the latter assets also provide information about $\alpha_{A}$ when they are mispriced by the benchmarks, i.e. when $\alpha_{N} \neq 0$. Our inclusion of the three industry portfolios is motivated primarily by the first consideration, explaining variance. Although we don't dismiss the possibility of their being mispriced, those portfolios are constructed to capture the most important sources of industry-related variation that is not accounted for by the other passive assets. On the other hand, our inclusion of CMS and MOM is motivated chiefly by the second consideration, mispricing. Evidence in other studies indicates that those spread positions may not be priced completely by the three benchmark

\footnotetext{
${ }^{5}$ Using up to 60 months of data through December of year $s-1$, the "pre-formation" HML betas are computed in a regression of the stock's excess returns on "fixed-weight" versions of the FF factors, which hold the weights on the constituent stocks constant at their June-end values of year $s$. Using the fixed-weight factors, as suggested by Daniel and Titman (1997), increases the dispersion in the "post-formation" betas of the resulting portfolios.
} 
factors, MKT, SMB, and HML. For example, Daniel and Titman (1997) conclude that, during the post-1963 period, characteristic-matched spreads in HML beta produce nonzero alphas with respect to the Fama-French three-factor model. ${ }^{6}$ Fama and French (1996) report a large three-factor alpha for the momentum strategy of Jegadeesh and Titman (1993). Of course, to be useful in estimating $\alpha_{A}, \mathrm{CMS}$ and MOM also have to explain additional variance of the fund's returns, and we find that to be the case for many funds.

Parsimony is a consideration limiting our number of non-benchmark assets to five. As discussed earlier, the degrees-of-freedom effect in finite samples argues against indiscriminately specifying a large number of non-benchmark assets. One might instead include a larger number of the characteristic-matched spreads, say one for each size/book-to-market subgroup, or include all 20 industry portfolios instead of constructing the smaller set of three. We tried such alternatives and found that they quite often produce estimates of $\alpha_{A}$ similar to those obtained using the smaller set of five, but the precision of the estimates based on the larger set is lower. The OLS estimators of $\delta_{A}$ and $c_{A N}$ are undefined, or essentially infinitely imprecise, when the total number of passive assets exceeds the length of the fund's history. The shrinkage estimator (explained below) can still be computed in that case, but it often yields a less precise inference than when fewer non-benchmark assets are used.

It is likely that future research could refine the selection of non-benchmark assets and further increase the precision of estimated alphas. A different set of non-benchmark assets could be specified for each fund, so that the assets have a high correlation with the specific fund at hand. With sector funds, for example, a passive index for the same sector could be included. A larger number of non-benchmark assets could be used for a fund with a longer history, since the degrees-of-freedom problem is then less severe. In general, some optimization over the set of non-benchmark assets would almost certainly increase the precision of our alpha estimates. Our specification of non-benchmark assets, motivated chiefly by simplicity, understates the potential gains from using non-benchmark assets to help estimate fund performance.

\footnotetext{
${ }^{6}$ Davis, Fama, and French (2000) find that a hypothesis of zero mispricing for such spreads cannot be rejected within the longer 1929-97 period. In response to a referee's concern that some of the characteristicrelated evidence could have been data-mined, we repeated our analysis with CMS excluded from the set of non-benchmark assets. The results are very close to those with CMS included.
} 


\subsection{The Bayesian framework}

We compute the posterior means and variances of both $\alpha_{A}$ and $S_{A}$. The posterior moments of $\alpha_{A}$ can be obtained analytically, whereas the moments of $S_{A}$ must be evaluated numerically be making repeated draws from the joint posterior distribution of the parameters. Derivations of the posterior moments of $\alpha_{A}$ as well as the details of computing the posterior moments of $S_{A}$ are provided in the Appendix. The stochastic setting and specification of prior beliefs is discussed below.

The regression disturbances in (4) and (5) are assumed to be normally distributed, independently and identically across $t$. Recall from an earlier discussion that the likelihood function for each fund can then be expressed as a product of two factors, one for each regression. We assume that the disturbances in (5) are uncorrelated across funds, which implies that the likelihood functions across funds are independent. ${ }^{7}$ Non-benchmark assets thus play yet another role, in that they account for covariance in fund returns that is not captured fully by the benchmarks. We also specify prior beliefs in which the parameters of the regression in (5) are independent across funds. The independence of the prior and the likelihood across funds allows us to conduct the analysis fund by fund.

First consider the parameters of the regression in (4). The prior distribution for $\Sigma$, the covariance matrix of $\epsilon_{N, t}$, is specified as inverted Wishart,

$$
\Sigma^{-1} \sim W\left(H^{-1}, \nu\right)
$$

We set the degrees of freedom $\nu=m+3$, so that the prior contains very little information about $\Sigma$. From the properties of the inverted Wishart distribution (e.g., Anderson (1984)), the prior expectation of $\Sigma$ equals $H /(\nu-m-1)$. We specify $H=s^{2}(\nu-m-1) I_{m}$, so that $\mathrm{E}(\Sigma)=s^{2} I_{m}$. Following an "empirical Bayes" approach, the value of $s^{2}$ is set equal to the average of the diagonal elements of the sample estimate of $\Sigma$ obtained using OLS. Conditional on $\Sigma$, the prior for $\alpha_{N}$ is specified as a normal distribution,

$$
\alpha_{N} \mid \Sigma \sim N\left(0, \sigma_{\alpha_{N}}^{2}\left(\frac{1}{s^{2}} \Sigma\right)\right)
$$

Pástor and Stambaugh (1999) introduce the same type of prior for a single element of $\alpha_{N}$, and Pástor (2000) and Pástor and Stambaugh (2000, 2001) apply the multivariate version in (17) to portfolio-choice problems. Having the conditional prior covariance matrix of $\alpha_{N}$ be

\footnotetext{
${ }^{7}$ Our mutual fund data, discussed in Section 3, are consistent with this assumption. The average correlation of the disturbances in (5), calculated across all pairs of funds whose return histories overlap for at least 12 months, is less than 0.02 .
} 
proportional to $\Sigma$ is motivated by the recognition that there exist portfolios of the passive assets with high Sharpe ratios if the elements of $\alpha_{N}$ are large when the elements of $\Sigma$ are small (MacKinlay, 1995.) Such combinations receive lower prior probabilities under (17) than when each element of $\alpha_{N}$ has standard deviation $\sigma_{\alpha_{N}}$ but is distributed independently of $\Sigma$. The prior distribution for $B_{N}$ is diffuse and independent of $\alpha_{N}$ and $\Sigma$.

Our earlier discussion focuses on the cases in which the benchmarks' ability to price the non-benchmark assets is assumed to be either perfect or nonexistent. That is, either $\alpha_{N}$ is set to the zero vector or the prior beliefs about $\alpha_{N}$ are diffuse. These two cases represent the opposite extremes on a continuum characterized by $\sigma_{\alpha_{N}}$, the marginal prior standard deviation of each element in $\alpha_{N}$. Specifying $\sigma_{\alpha_{N}}=0$ is equivalent to setting $\alpha_{N}=0$, corresponding to perfect confidence in the benchmarks' pricing ability. A diffuse prior for $\alpha_{N}$ corresponds to $\sigma_{\alpha_{N}}=\infty$. With a nonzero finite value of $\sigma_{\alpha_{N}}$, prior beliefs are centered on the pricing restriction, but some degree of mispricing is entertained. We refer to $\sigma_{\alpha_{N}}$ as "mispricing uncertainty."

Next consider the parameters of the regression in (5). The prior for $\sigma_{u}^{2}$, the variance of $u_{A, t}$, is specified as inverted gamma, or

$$
\sigma_{u}^{2} \sim \frac{\nu_{0} s_{0}^{2}}{\chi_{\nu_{0}}^{2}}
$$

where $\chi_{\nu_{0}}^{2}$ denotes a chi-square variate with $\nu_{0}$ degrees of freedom. Define $c_{A}=\left(c_{A N}^{\prime} c_{A B}^{\prime}\right)^{\prime}$. Conditional on $\sigma_{u}^{2}$, the priors for $\delta_{A}$ and $c_{A}$ are specified as normal distributions, independent of each other:

$$
\delta_{A} \mid \sigma_{u}^{2} \sim N\left(\delta_{0},\left(\frac{\sigma_{u}^{2}}{\mathrm{E}\left(\sigma_{u}^{2}\right)}\right) \sigma_{\delta}^{2}\right)
$$

and

$$
c_{A} \mid \sigma_{u}^{2} \sim N\left(c_{0},\left(\frac{\sigma_{u}^{2}}{\mathrm{E}\left(\sigma_{u}^{2}\right)}\right) \Phi_{c}\right) .
$$

The marginal prior variance of $\delta_{A}$ is $\sigma_{\delta}^{2}$, and the marginal prior covariance matrix of $c_{A}$ is $\Phi_{c}$. We set $\sigma_{\delta}^{2}=\infty$ (or, computationally, a very large value), which implies that the prior for $\alpha_{A}$ is diffuse and that the prior mean $\delta_{0}$ is irrelevant. ${ }^{8}$

Values for $s_{0}, \nu_{0}, c_{0}$, and $\Phi_{c}$ in (18) through (20) are specified using an empirical-Bayes procedure. The basic idea is that a given fund is viewed as a draw from a cross-section of funds with the same investment objective, so the prior uncertainty about a parameter for the fund is governed by the cross-sectional dispersion of that parameter. The empirical-Bayes

\footnotetext{
${ }^{8}$ In an analysis of mutual-fund investment, Pástor and Stambaugh (2001) set $\sigma_{\delta}^{2}$ to finite values and specify $\delta_{0}$ to reflect a fund's costs.
} 
procedure uses the data to infer those properties of the cross-section. The prior mean and covariance matrix of $c_{A}$, denoted by $c_{0}$ and $\Phi_{c}$, are set equal to the corresponding sample cross-sectional moments of $\hat{c}_{A}$, the OLS estimate of $c_{A}$, for all funds with at least 60 months of data and the same investment objective as the fund at hand. (The investment objectives are displayed in Table 1.) Setting $\Phi_{c}$ equal to the sample covariance matrix of $\hat{c}_{A}$, without adjusting for the sampling variation in those estimates, overstates the dispersion across funds in the true values of $c_{A}$. In that sense, our use of this empirical-Bayes procedure is conservative, in that it applies an intentionally modest degree of shrinkage toward the crosssectional mean of $\hat{c}_{A}$ when computing the posterior moments of $c_{A}$ for a given fund. With a diffuse prior on $c_{A}$, or no shrinkage, the estimator (posterior mean) of $c_{A}$ is simply the OLS value $\hat{c}_{A}$. The degree of shrinkage applied here, albeit conservative, gives a more precise estimator of $c_{A}$, especially for a short-history fund, and thereby allows the non-benchmark assets to reveal more of their information about the fund's alpha.

The inverted gamma prior density for $\sigma_{u}^{2}$ implies (e.g., Zellner (1971, p. 372)),

$$
\mathrm{E}\left(\sigma_{u}^{2}\right)=\frac{\nu_{0} s_{0}^{2}}{\nu_{0}-2},
$$

and

$$
\nu_{0}=4+\frac{2\left(\mathrm{E}\left(\sigma_{u}^{2}\right)\right)^{2}}{\operatorname{Var}\left(\sigma_{u}^{2}\right)} .
$$

We substitute the cross-sectional mean and variance of $\hat{\sigma}_{u}^{2}$ for $\mathrm{E}\left(\sigma_{u}^{2}\right)$ and $\operatorname{Var}\left(\sigma_{u}^{2}\right)$ in $(21)$ and (22). The value of $\nu_{0}$ is set to the next largest integer of the resulting value on the right-hand side of (22), and then that value of $\nu_{0}$ implies the value of $s_{0}^{2}$ using (21). Here again, using the cross-sectional variance of $\hat{\sigma}_{u}^{2}$ without adjusting for sampling error produces a conservative amount of shrinkage toward the cross-sectional mean of $\hat{\sigma}_{u}^{2}$ for funds with the same objective.

Our framework assumes fund managers have no ability to time the benchmark or nonbenchmark assets. More generally, our framework models a fund's sensitivities to passive assets as constant over time. One way of relaxing this assumption is to model these coefficients as linear functions of state variables, as suggested by Shanken (1990) and applied by Ferson and Schadt (1996) in a mutual-fund context. In such a modification, passive asset returns scaled by the state variables can be viewed as returns on additional passive assets (dynamic passive strategies). The GMM formulation in (14) easily accommodates scaled returns, and the Bayesian approach developed here could be extended to such a setting as well. Another approach to dealing with temporal variation in parameters could employ data on fund holdings. Daniel, Grinblatt, Titman, and Wermers (1997) and Wermers (2000), for example, use such data in characteristic-based studies of fund performance. 


\section{Empirical Analysis}

The mutual-fund data come from the 1998 CRSP Survivor Bias Free Mutual Fund Database. ${ }^{9}$ Our sample contains 2,609 domestic equity mutual funds with more than a year of available returns. Three quarters of our funds are still alive at the end of 1998. The funds are assigned to one of seven broad investment objectives, as described in the Appendix. Table 1 lists the number of funds in each objective, further classifying funds within each objective by the number of months in the fund's available return history. For each fund we compute the monthly return in excess of that on a one-month Treasury bill.

\subsection{Estimates of funds' alphas.}

Table 2 reports medians, within various fund classifications, of CAPM alphas (Panel A) and Fama-French alphas (Panel B). The posterior mean of $\alpha_{A}$, denoted as $\tilde{\alpha}_{A}$, is computed for $\sigma_{\alpha_{N}}$ equal to zero, two percent (annualized), and infinity. Recall that the usual OLS estimator, denoted as $\hat{\alpha}_{A}$, makes no use of seemingly unrelated non-benchmark assets. Also reported are median absolute differences between $\hat{\alpha}_{A}$ and $\tilde{\alpha}_{A}$. Not surprisingly, non-benchmark assets play a greater role in the estimation of CAPM alphas, since two of the non-benchmark assets in that case, SMB and HML, are already included as benchmarks when estimating FamaFrench alphas. Across all funds, the median value of $\left|\hat{\alpha}_{A}-\tilde{\alpha}_{A}\right|$ is two percent per annum for CAPM alphas but about one percent or less, depending on $\sigma_{\alpha_{N}}$, for Fama-French alphas. Note also that $\left|\hat{\alpha}_{A}-\tilde{\alpha}_{A}\right|$ is typically smaller for the funds with longer histories. With a longer history, $\hat{\alpha}_{A}$ becomes more precise, so the additional information in non-benchmark returns has a smaller impact.

The manner by which non-benchmark assets provide information is illustrated most dramatically in the case of CAPM alphas for small-company growth funds. For such funds, incorporating the information in non-benchmark assets typically makes a difference of between $7.2 \%$ and $8.3 \%$ per annum when estimating the CAPM alpha, depending on $\sigma_{\alpha_{N}}$. Nearly half of those 413 funds have track records of three years or less (see Table 1), and the bulk of their track records fall toward the end of the overall period. In recent years, small-firm indexes have underperformed their CAPM predictions, which is relevant when $\sigma_{\alpha_{N}}=0$, and they have also underperformed their long-run historical averages, which is relevant when $\sigma_{\alpha_{N}}=\infty$. (Both statements are relevant when $\sigma_{\alpha_{N}}=2 \%$.) Incorporating that

\footnotetext{
${ }^{9}$ CRSP, Center for Research in Security Prices, Graduate School of Business, The University of Chicago 1999, crsp.com. Used with permission. All rights reserved.
} 
information is accomplished in either case largely by including as a non-benchmark asset the size factor SMB, which is positively correlated with the small-company growth funds' non-market-related returns.

An important issue in performance evaluation is whether the mutual fund industry adds value beyond standard passive benchmarks. We address this issue by computing posterior probabilities that average fund alphas within various fund classifications are negative. These probabilities are computed based on 100,000 draws of the average alpha from its posterior distribution. The probabilities are reported in Table 3, together with posterior means of average CAPM alphas (Panel A) and Fama-French alphas (Panel B). Some differences between the average alphas in Table 3 and the median alphas in Table 2 reflect skewness in the cross-sectional distribution of fund alphas. For example, the average of the OLS estimates of the CAPM alphas across all funds is $-3.83 \%$, compared to their median of $-2.13 \%$.

With few exceptions, Table 3 supports the inference that average fund alphas are negative. For example, for each investment objective and each age group, the average of the OLS estimates of the CAPM alphas is negative with $100 \%$ probability. The averages of the OLS estimates of the Fama-French alphas are mostly negative, although they are reliably positive for funds with histories longer than 10 years. When the non-benchmark assets are included, the average performance across all funds remains significantly negative, although the performance of long-history funds and aggressive growth funds improves with skeptical prior beliefs about pricing $\left(\sigma_{\alpha_{N}}=\infty\right)$. The importance of beliefs about pricing can be illustrated by the average Fama-French alpha for small-cap growth funds. When the nonbenchmark assets are not used, there is a $50 \%$ probability that the average alpha for those funds is negative. When the non-benchmark assets are included, the probability that the average alpha is negative rises to $100 \%$ when those assets are believed to be exactly priced by the benchmarks, but it drops to $9 \%$ when no pricing relation is used.

Our universe of funds includes those that ceased existence before the end of the overall sample period in December 1998 (a quarter of our funds). The alpha estimates in Tables 2 and 3 are generally higher for funds with longer histories. This age-related pattern is not surprising, since funds with poor track records are less likely to be long lived. One might therefore ask whether the estimate of a fund's alpha should be adjusted according to whether or not it survived to the end of the sample. The posterior mean of alpha is conditioned on the returns of the fund and the passive assets. If one assumes that, conditional on those realized returns, the probability of survival is unaffected by conditioning on the true parameters as well, then conditioning on survival has no incremental effect on the posterior mean of the 
fund's alpha. This observation is made by Baks, Metrick and Wachter (2001), and their assumption that realized returns determine survival probabilities seems reasonable. ${ }^{10}$ For this reason, a survival-based adjustment to our estimates is unnecessary.

When interpreting the age-related patterns in Tables 2 and 3, recall that our estimates are based on non-informative prior beliefs, so as to make our approach comparable to the standard practice in which a fund's performance measure is computed without an adjustment for prior beliefs about reasonable values. When the prior is non-informative, poor performance, whether or not it contributed to a fund's death, is translated fully to an inference that the fund's alpha is low. Readers who think that our estimated alphas for the short-history funds seem too low essentially have informative prior beliefs, which is a reasonable alternative. For example, with a prior that each fund's alpha is drawn from a distribution with a finite variance and a common mean, the alphas of short-history funds are shrunk more toward the grand mean than the alphas of long-history funds, thereby reducing the age-related differences. (The prior exerts more influence in a shorter sample.) That shrinkage is not an adjustment for survival, however. By the earlier argument, no such adjustment is necessary, whether or not the prior is informative.

To investigate whether including the non-benchmark assets leads to a more precise inference about a fund's alpha, in Table 4 we examine the ratio of two posterior variances. The numerator of the ratio is the posterior variance of $\alpha_{A}$ under our model in which nonbenchmark assets are used and the prior variance for the elements of $\alpha_{N}$ is as given in the column heading. Recall that the posterior mean of $\alpha_{A}$ in that case is denoted as $\tilde{\alpha}_{A}$. The denominator of the ratio is the posterior variance of $\alpha_{A}$ when the non-benchmark assets are not used and diffuse priors are assigned to all parameters. The posterior mean of $\alpha_{A}$ in that case is the OLS estimate $\hat{\alpha}_{A}$. For ease of discussion, we commit a slight abuse of notation and refer to the posterior variances in the numerator and denominator as the "variances" of

\footnotetext{
${ }^{10}$ Let $R$ denote the returns over a given period, let $s$ denote a random variable indicating whether or not a given fund dies at the end of that period, and let $\theta$ denote the vector of true (unknown) parameters of the return distribution. As observed by Baks, Metrick, and Wachter (2001), the assumption

$$
p(s \mid R, \theta)=p(s \mid R)
$$

implies, using Bayes' rule, that$$
p(\theta \mid R, s)=\frac{p(s \mid R, \theta) p(\theta \mid R)}{p(s \mid R)}=p(\theta \mid R) .
$$

Our assumption of independence across funds of the disturbances in (5) permits a fund-by-fund treatment, as discussed earlier, so the above holds when $R$ includes the returns on all funds in our universe. Therefore, a fund's survival probability can depend not only on its performance relative to benchmarks but relative to other funds as well.
} 
$\tilde{\alpha}_{A}$ and $\hat{\alpha}_{A}$. These variances reflect the precision of inferences about $\alpha_{A}$ in the sense generally associated with standard errors in a frequentist setting. In fact, the denominator of the ratio equals the squared standard error computed in the usual regression model.

For most funds, a more precise inference about alpha is obtained by including nonbenchmark assets. Table 4 reports the median ratio of the variance of $\tilde{\alpha}_{A}$ to the variance of $\hat{\alpha}_{A}$. Also reported is the fraction of those ratios that are less than one. For the CAPM alpha estimates, the median variance ratio across all funds is approximately 0.7 , and the ratio is less than one for roughly $90 \%$ of the funds. For Fama-French alphas, the ratio has a median of about 0.85 and is less than one for roughly $80 \%$ of the funds. In general, the median ratio is higher for the funds with longer histories. Note from Table 1 that funds with track records of at least 20 years represent only about $5 \%$ or our sample (139 out of 2609). For those funds, the OLS estimates of Fama-French alphas are typically about as precise as the estimates that incorporate the non-benchmark assets: the median variance ratios are 1.00 or just slightly less, and the ratios cluster fairly tightly around that value. At the other extreme lies the variance ratio associated with estimating CAPM alphas for small-company growth funds. That variance ratio has a median between 0.33 and 0.39 , depending on $\sigma_{\alpha_{N}}$, and the ratio is less than one for all such funds in our sample. Thus, for small-company growth funds in particular, not only is the CAPM $\tilde{\alpha}_{A}$ substantially higher than the CAPM $\hat{\alpha}_{A}$, it is also substantially more precise.

Recall that estimates of $\alpha_{A}$ are identical across different specifications of the benchmarks when one assumes the non-benchmark assets are priced exactly under each specification. In Table 2, note that the median values of $\tilde{\alpha}_{A}$ are indeed the same in Panels A and B when $\sigma_{\alpha_{N}}=0$ (which sets $\alpha_{N}=0$ ). Table 5 compares estimates of CAPM and Fama-French alphas when $\sigma_{\alpha_{N}}=2 \%$ (Panel A) and $\sigma_{\alpha_{N}}=\infty$ (Panel B). As expected, the median absolute differences between models are typically larger in the second case, but those differences are still substantially less than the median absolute differences between OLS estimates (Panel C). In other words, even when the non-benchmark assets are not believed to be priced whatsoever by either model's benchmarks, their presence in the estimation still makes the definition of $\alpha_{A}$ substantially less important than when they are not used at all. Across all funds, the median absolute difference between estimated CAPM and Fama-French alphas is $0.42 \%$ (per annum) under $\sigma_{\alpha_{N}}=2 \%$ and $1.24 \%$ under $\sigma_{\alpha_{N}}=\infty$, as compared to $2.28 \%$ for the OLS estimates. For small-company growth funds, the median difference is $0.69 \%$ under $\sigma_{\alpha_{N}}=2 \%$ and $2.03 \%$ under $\sigma_{\alpha_{N}}=\infty$, as compared to $8.07 \%$ for the OLS estimates.

Table 6 compares alphas defined for a given set of benchmarks and estimated with or 
without the pricing restriction imposed on the non-benchmark assets. That is, for $\alpha_{A}$ defined with respect to a given pricing model, we compare estimates under $\sigma_{\alpha_{N}}=0$ to estimates under $\sigma_{\alpha_{N}}=\infty$. Across all funds, the median difference is $0.94 \%$ for CAPM alphas and $0.68 \%$ for Fama-French alphas. Interestingly, the median differences display little if any relation to the length of the fund's history. Most of the median differences in the two-way sort (by objective and age) are $2 \%$ or less, with the exception of sector funds. For the funds with the longest histories, the effect of imposing the pricing restriction on the non-benchmark assets is often as large as the effect of not including the non-benchmark assets at all (shown earlier in Table 2). The latter effect is more important for history lengths of ten years or less when estimating CAPM alphas and five years or less when estimating Fama-French alphas. Note from Table 1 that about $85 \%$ of the equity funds in our sample have history lengths less than 10 years, and about $60 \%$ have histories of five years or less.

\subsection{Estimates of funds' Sharpe ratios.}

Table 7 reports medians of funds' Sharpe ratios, within various fund classifications, in a manner analogous to Table 2 for funds' alphas. The usual sample estimate of a fund's Sharpe ratio $S_{A}$, denoted as $\hat{S}_{A}$, is computed using only the fund's excess returns. In contrast, the posterior mean of $S_{A}$, denoted as $\tilde{S}_{A}$, also reflects information in returns of seemingly unrelated assets. The posterior means of $S_{A}$ for the CAPM and the Fama-French model are reported for prior beliefs in which the pricing relations are assumed to hold exactly $\left(\sigma_{\alpha_{N}}=0\right)$. For the "No Model" results, prior beliefs in any pricing model are diffuse $\left(\sigma_{\alpha_{N}}=\infty\right)$.

The median value of $\hat{S}_{A}$ across all funds is 0.68 , but the median value of $\tilde{S}_{A}$ is only about half of that, ranging from 0.31 for the CAPM to 0.34 for "No Model". Since a typical fund in our sample has been around only during the bull market in the 1990s, the median $\hat{S}_{A}$ is quite high. The values of $\tilde{S}_{A}$ reflect additional information in the longer histories of returns on seemingly unrelated assets. Those returns are on average lower than the returns in the 1990s, resulting in lower values for $\tilde{S}_{A}$. Also note that $\tilde{S}_{A}$ is not very sensitive to the choice of the pricing model. The model benchmarks are not used to define the Sharpe ratio; the only role for the model is to determine the long-run expected returns on the non-benchmark assets. When estimating Sharpe ratios, including the long histories of seemingly unrelated assets is clearly more important than choosing a pricing model.

Table 7 also reports the median absolute differences between $\hat{S}_{A}$ and $\tilde{S}_{A}$. The median

value of $\left|\hat{S}_{A}-\tilde{S}_{A}\right|$ across all funds is large, about 0.4 . The median absolute differences are 
the smallest for the longest-history funds, because, with a longer history, $\hat{S}_{A}$ becomes more precise and any additional information has a smaller effect. The long histories of returns on seemingly unrelated passive assets clearly contain a lot of information about the funds' Sharpe ratios, especially (but not exclusively) for funds with relatively short track records.

We examine the precision of the estimated Sharpe ratios in the same manner as reported previously in Table 4 for the precision of alphas. Table 8 reports the median ratio of the posterior variances of $\tilde{S}_{A}$ and $\hat{S}_{A}$ as well as the fraction of those ratios that are less than one. The numerator of the ratio is the posterior variance of $S_{A}$ under our model in which returns on all passive assets are used, with prior beliefs corresponding either to exact pricing (CAPM, Fama-French models) or to no pricing ("No Model"). This posterior variance is computed from a large number of independent draws of each fund's Sharpe ratio from its posterior distribution, as described in the Appendix. The denominator of the ratio is the posterior variance of $S_{A}$ when only the fund returns are used and diffuse priors are assigned to all parameters.

The results in Table 8 indicate that seemingly unrelated assets provide more precise Sharpe ratio estimates for over $99 \%$ of the funds in our universe. The median of the variance ratios across all funds is about 0.23 , with little dependence on the choice of the pricing model. The precision of our Sharpe ratio estimates is therefore four to five times higher on average than the precision of the usual estimates. The median variance ratios increase uniformly with funds' history lengths, from about 0.16 for the funds with less than two years of data to about 0.88 for the funds with at least twenty years of data. Comparing the results in Table 8 to those in Table 4 reveals that seemingly unrelated assets help increase the precision of the funds' Sharpe ratios even more than the precision of their alphas. For Sharpe ratios, the set of seemingly unrelated assets includes all passive assets, not only non-benchmark assets, so the additional information contributed by seemingly unrelated assets is even more important.

Morningstar, Inc., a leading provider of mutual fund information, ranks funds into five categories (one to five stars) based on a risk-adjusted rating in which a measure of the fund's downside volatility is subtracted from a measure of the fund's average excess return. Although the Sharpe ratio and the Morningstar rating are defined differently, they share the same basic risk-adjustment concept and often provide similar rankings of funds, as demonstrated by Sharpe $(1997,1998)$. Moreover, the principles underlying the increase in precision in our Sharpe ratio estimates can clearly be used to improve the precision of Morningstar's 
risk-adjusted ratings, especially the component based on the fund's average-excess return. ${ }^{11}$ Since Morningstar rankings are known to be influential to mutual-fund investors, an important question is whether the fund rankings are affected by including seemingly unrelated assets. To explore this question, we examine rankings based on estimated Sharpe ratios. Specifically, we sort all 1,585 funds with at least three years of data into five categories, using the same breakpoints as Morningstar (top 10\%, next 22.5\%, next 35\%, next 22.5\%, bottom $10 \%$ ). The funds are ranked first by their $\hat{S}_{A}$ 's and then separately by their $\tilde{S}_{A}$ 's, with the latter computed using prior beliefs that are noninformative about pricing.

Table 9 reports, in a five-by-five matrix, the numbers of funds in the intersection of the two independent rankings. For example, the $(1,3)$ cell contains the number of funds that are ranked in the top group based on $\tilde{S}_{A}$ and in the third group based on $\hat{S}_{A}$. Large off-diagonal numbers in the matrix reveal that the $\tilde{S}_{A}$ ranking is quite different from the $\hat{S}_{A}$ ranking. At the extreme, there are three funds that are classified in the bottom $10 \%$ using the traditional $\hat{S}_{A}$ but in the top $10 \%$ using the more precise $\tilde{S}_{A}$. Only 526 out of the 1,585 funds are ranked in the same category under both $\hat{S}_{A}$ and $\tilde{S}_{A}$, and the rank correlation is only 0.53 . With informative prior beliefs about pricing, the differences between the two rankings are even slightly larger. Clearly, the information in returns on seemingly unrelated assets has an important impact on fund rankings.

\section{Conclusions}

This study develops and applies methods for improved estimation of performance measures using information in returns on "seemingly unrelated" assets not used to define those measures. A fund's alpha is defined relative to a set of passive benchmarks. The typically reported OLS estimate of alpha ignores information provided by returns on non-benchmark assets. The non-benchmark assets help estimate alpha if they are priced to some extent by the benchmarks or if their return histories are longer than the fund's. A fund's Sharpe ratio can be estimated more precisely by using information in returns on assets other than just the fund.

Using a sample of 2,609 U.S. equity mutual funds, we demonstrate that the returns on seemingly unrelated assets contain substantial information about fund performance. For

\footnotetext{
${ }^{11}$ Whenever the average excess return on the fund's broad investment class is positive, the Morningstar rating uses essentially the same average excess fund return generally used to compute the fund's Sharpe ratio.
} 
most funds, the estimates of alpha that incorporate the information in non-benchmark returns are more precise than the standard estimates. In the case of small-company growth funds, for example, the non-benchmark assets allow alphas defined with respect to the market benchmark to be estimated with only one third of the variance associated with the usual OLS estimates of those alphas. Across all funds, Sharpe ratios estimated using seemingly unrelated assets have a median only about half that of the usual estimates and are generally four to five times more precise. Moreover, fund rankings based on the improved Sharpe-ratio estimates differ substantially from those based on the usual estimates.

Compared to the usual estimates, the estimates of alpha that incorporate the information in the non-benchmark assets tend to exhibit less variation across different specifications of the benchmarks. At the extreme, if one believes that some subset of the passive assets used in the estimation prices the other passive assets exactly, then the estimate of alpha is the same regardless of which subset is designated as the benchmarks that define alpha. In other words, if one believes dogmatically in a pricing model, it does not matter which model that is when estimating alpha. For most funds, we find that including information in non-benchmark assets is more important than specifying the degree to which the non-benchmark assets are priced by the benchmarks. We also find that, across different beliefs about pricing, most funds have underperformed the CAPM and Fama-French benchmarks. 


\section{Appendix}

This Appendix derives the posterior moments of $\alpha_{A}$ and $S_{A}$. The prior for the parameters of the regression in (5) is independent of the prior for the parameters of the regression in (4). In addition, both of those priors are assumed to be independent of the (diffuse) prior for $E_{B}$ and $V_{B B}$, the mean and covariance matrix of the normal distribution for $r_{B, t}$. The independence of the priors and the independence of $u_{A, t}$ and $\epsilon_{N, t}$ imply that the posterior distributions for the parameters of the regression in (4) depend only on the sample of passive asset returns, not the fund returns. We first provide the moments of those distributions, relying on the derivations in Pástor and Stambaugh (2000), henceforth referred to as PS. Those moments are then combined with the posterior moments of $\delta_{A}$ and $c_{A}$ to obtain the posterior moments of $\alpha_{A}$. The posterior distribution of $\alpha_{N}$ is independent of $\delta_{A}$ and $c_{A N}$, so the posterior mean of $\alpha_{A}$ is obtained simply by evaluating the right-hand side of (7) at the posterior means of $\alpha_{N}, \delta_{A}$ and $c_{A N}$. The posterior variances of $\alpha_{A}$ can also be computed using the posterior first and second moments of $\alpha_{N}, \delta_{A}$ and $c_{A N}$. These results are then used to compute numerically, using simulation, the posterior moments of $S_{A}$. The Appendix concludes with an explanation of how funds are classified by investment objectives.

\section{A.1. Posterior distribution for the passive-asset parameters}

Define $Y=\left(r_{N, 1}, \ldots, r_{N, T}\right)^{\prime}, X=\left(r_{B, 1}, \ldots, r_{B, T}\right)^{\prime}$, and $Z=\left(\iota_{T} X\right)$, where $\iota_{T}$ denotes a $T$-vector of ones. Also define the $(k+1) \times m$ matrix $G=\left(\alpha_{N} B_{N}\right)^{\prime}$, and let $g=\operatorname{vec}(G)$. For the $T$ observations $t=1, \ldots, T$, the regression model in (4) can be written as

$$
Y=Z G+U, \quad \operatorname{vec}(U) \sim N\left(0, \Sigma \otimes I_{T}\right),
$$

where $U=\left(\epsilon_{N, t}, \ldots, \epsilon_{N, T}\right)^{\prime}$. Define the statistics $\hat{G}=\left(Z^{\prime} Z\right)^{-1} Z^{\prime} Y, \hat{g}=\operatorname{vec}(\hat{G}), \hat{\Sigma}=$ $(Y-Z \hat{G})^{\prime}(Y-Z \hat{G}) / T, \hat{E}_{B}=X^{\prime} \iota_{T} / T$, and $\hat{V}_{B B}=\left(X-\iota_{T} \hat{E}_{B}^{\prime}\right)^{\prime}\left(X-\iota_{T} \hat{E}_{B}^{\prime}\right) / T$. Let $\theta_{P}$ denote the parameters of the joint distribution of the passive asset returns $\left(G, \Sigma, E_{B}\right.$, and $\left.V_{B B}\right)$, and define the $T \times p$ sample matrix of passive returns, $R_{P}=(X Y)$. The likelihood function for the passive returns can be factored as

$$
p\left(R_{P} \mid \theta_{P}\right)=p\left(Y \mid \theta_{P}, X\right) p\left(X \mid \theta_{P}\right)
$$

where

$$
\begin{aligned}
p\left(Y \mid \theta_{P}, X\right) & \propto|\Sigma|^{-\frac{T}{2}} \exp \left\{-\frac{1}{2} \operatorname{tr}(Y-Z G)^{\prime}(Y-Z G) \Sigma^{-1}\right\} \\
p\left(X \mid \theta_{P}\right) & \propto\left|V_{B B}\right|^{-\frac{T}{2}} \exp \left\{-\frac{1}{2} \operatorname{tr}\left(X-\iota_{T} E_{B}^{\prime}\right)^{\prime}\left(X-\iota_{T} E_{B}^{\prime}\right) V_{B B}^{-1}\right\} .
\end{aligned}
$$


The joint prior distribution of all passive-return parameters is

$$
p\left(\theta_{P}\right)=p\left(\alpha_{N} \mid \Sigma\right) p(\Sigma) p\left(B_{N}\right) p\left(E_{B}\right) p\left(V_{B B}\right)
$$

where

$$
\begin{aligned}
p\left(\alpha_{N} \mid \Sigma\right) & \propto|\Sigma|^{-\frac{1}{2}} \exp \left\{-\frac{1}{2} \alpha_{N}^{\prime}\left(\frac{\sigma_{\alpha_{N}}^{2}}{s^{2}} \Sigma\right)^{-1} \alpha_{N}\right\} \\
p(\Sigma) & \propto|\Sigma|^{-\frac{\nu+m+1}{2}} \exp \left\{-\frac{1}{2} \operatorname{tr} H \Sigma^{-1}\right\} \\
p\left(B_{N}\right) & \propto 1 \\
p\left(E_{B}\right) & \propto 1 \\
p\left(V_{B B}\right) & \propto\left|V_{B B}\right|^{-\frac{k+1}{2}} .
\end{aligned}
$$

The priors of $B_{N}, E_{B}$, and $V_{B B}$ are diffuse. The prior of $\Sigma$ is inverted Wishart with a small number of degrees of freedom, so that it is essentially noninformative. The prior on $\alpha_{N}$ given $\Sigma$ is normal and centered at the pricing restriction. Let $\mathrm{D}$ denote a $(k+1) \times(k+1)$ matrix whose $(1,1)$ element is $\frac{s^{2}}{\sigma_{\alpha_{N}}^{2}}$ and all other elements are zero. Also let $F=D+Z^{\prime} Z$ and $Q=Z^{\prime}\left(I_{T}-Z F^{-1} Z^{\prime}\right) Z$. Applying the analysis in PS, the likelihood and the prior are combined to obtain the posteriors of the parameters of interest. The posterior distributions of the slopes and the residual covariance matrix from regression (A.1) are

$$
\begin{aligned}
g \mid \Sigma, R_{P} & \sim N\left(\tilde{g}, \Sigma \otimes F^{-1}\right) \\
\Sigma^{-1} \mid R_{P} & \sim W\left(\left(H+T \hat{\Sigma}+\hat{G}^{\prime} Q \hat{G}\right)^{-1}, T+\nu-k\right),
\end{aligned}
$$

where $W$ denotes the Wishart distribution, and the posterior moments are

$$
\begin{aligned}
\tilde{g} & =\mathrm{E}\left(g \mid R_{P}\right)=\left(I_{m} \otimes F^{-1} Z^{\prime} Z\right) \hat{g} \\
\tilde{\Sigma} & =\mathrm{E}\left(\Sigma \mid R_{P}\right)=\frac{1}{T+\nu-m-k-1}\left(H+T \hat{\Sigma}+\hat{G}^{\prime} Q \hat{G}\right) \\
\operatorname{Var}\left(g \mid R_{P}\right) & =\tilde{\Sigma} \otimes F^{-1} .
\end{aligned}
$$

The posteriors of the mean and the covariance matrix of the benchmark returns are

$$
\begin{aligned}
E_{B} \mid V_{B B}, R_{P} & \sim N\left(\hat{E}_{B}, \frac{1}{T} V_{B B}\right) \\
V_{B B}^{-1} \mid R_{P} & \sim W\left(\left(T \hat{V}_{B B}\right)^{-1}, T-1\right),
\end{aligned}
$$

and the posterior moments are

$$
\begin{aligned}
\tilde{E}_{B} & =\mathrm{E}\left(E_{B} \mid R_{P}\right)=\hat{E}_{B} \\
\tilde{V}_{B B} & =\mathrm{E}\left(V_{B B} \mid R_{P}\right)=\frac{T}{T-k-2} \hat{V}_{B B} \\
\operatorname{Var}\left(E_{B} \mid R_{P}\right) & =\frac{1}{T-k-2} \hat{V}_{B B} .
\end{aligned}
$$

Posterior means are denoted using tildes for the remainder of the Appendix. 


\section{A.2. Posterior distribution for the parameters of (5)}

Let $r_{A}(S \times 1)$ contain $S$ observations of $r_{A, t}$, the return on a given fund A. We assume $S \leq T$ and that the (consecutive) months in which $r_{A, t}$ is observed form a subset of those in which $r_{N, t}$ and $r_{B, t}$ are observed. The assumption that the disturbances in (5) are independent across funds, coupled with the assumption that the priors of that regression's parameters are independent across funds, implies that the posterior distribution for a given fund's parameters of the regression in (5) does not depend on the observed returns of the other funds (conditional on the passive return sample $R_{P}$ ). Let $\theta_{A}$ denote the set of parameters $\delta_{A}, c_{A}$, and $\sigma_{u}^{2}$. Our various modeling assumptions give

$$
\begin{aligned}
p\left(\theta_{A}, \theta_{P} \mid R_{P}, r_{A}\right) & \propto p\left(\theta_{A}, \theta_{P}\right) p\left(R_{P}, r_{A} \mid \theta_{A}, \theta_{P}\right) \\
& =p\left(\theta_{A}\right) p\left(\theta_{P}\right) p\left(r_{A} \mid R_{P}, \theta_{A}, \theta_{P}\right) p\left(R_{P} \mid \theta_{A}, \theta_{P}\right) \\
& =p\left(\theta_{A}\right) p\left(r_{A} \mid R_{P}, \theta_{A}\right) p\left(\theta_{P}\right) p\left(R_{P} \mid \theta_{P}\right) \\
& \propto p\left(\theta_{A} \mid R_{P}, r_{A}\right) p\left(\theta_{P} \mid R_{P}\right) .
\end{aligned}
$$

The second factor in (A.21) is the posterior derived previously. The first factor, the posterior for $\theta_{A}$, combines the priors given in (18) through (20) with the likelihood,

$$
p\left(r_{A} \mid R_{P}, \theta_{A}\right) \propto \frac{1}{\sigma_{u}^{T}} \exp \left\{-\frac{1}{2 \sigma_{u}^{2}}\left(r_{A}-Z_{A} \phi_{A}\right)^{\prime}\left(r_{A}-Z_{A} \phi_{A}\right)\right\},
$$

where $R_{P, A}$ denotes the $S$ rows of $R_{P}$ corresponding to the months in which $r_{A, t}$ is observed, $Z_{A}=\left(\iota_{S} R_{P, A}\right)$, and $\phi_{A}=\left(\delta_{A} c_{A}^{\prime}\right)^{\prime}$. The prior densities corresponding to (18) through (20) are given by

$$
p\left(\sigma_{u}\right) \propto \frac{1}{\sigma_{u}^{\nu_{0}+1}} \exp \left\{-\frac{\nu_{0} s_{0}^{2}}{2 \sigma_{u}^{2}}\right\}
$$

and

$$
p\left(\phi_{A} \mid \sigma_{u}\right) \propto \frac{1}{\sigma_{u}^{p+1}} \exp \left\{-\frac{1}{2 \sigma_{u}^{2}}\left(\phi_{A}-\phi_{0}\right)^{\prime} \Lambda_{0}\left(\phi_{A}-\phi_{0}\right)\right\},
$$

where $\phi_{0}=\left(\delta_{0} c_{0}^{\prime}\right)^{\prime}$ and

$$
\Lambda_{0}=\left(\frac{\nu_{0} s_{0}^{2}}{\nu_{0}-2}\right)\left[\begin{array}{cc}
\sigma_{\delta}^{2} & 0 \\
0 & \Phi_{c}
\end{array}\right]^{-1}
$$

The product of (A.22), (A.23), and (A.24) gives, after simplifying, ${ }^{12}$

$$
\begin{aligned}
p\left(\phi_{A}, \sigma_{u} \mid R_{P}, r_{A}\right) \propto & \frac{1}{\sigma_{u}^{p+1}} \exp \left\{-\frac{1}{2 \sigma_{u}^{2}}\left(\phi_{A}-\tilde{\phi}_{A}\right)^{\prime}\left(\Lambda_{0}+Z_{A}^{\prime} Z_{A}\right)\left(\phi_{A}-\tilde{\phi}_{A}\right)\right\} \\
& \times \frac{1}{\sigma_{u}^{T+\nu_{0}+1}} \exp \left\{-\frac{h_{A}}{2 \sigma_{u}^{2}}\right\},
\end{aligned}
$$

\footnotetext{
${ }^{12}$ See Zellner (1971, pp. $75-76$ ) for a similar derivation.
} 
where

$$
\begin{aligned}
\tilde{\phi}_{A} & =\left(\Lambda_{0}+Z_{A}^{\prime} Z_{A}\right)^{-1}\left(\Lambda_{0} \phi_{0}+Z_{A}^{\prime} r_{A}\right) \\
h_{A} & =\nu_{0} s_{0}^{2}+r_{A}^{\prime} r_{A}+\phi_{0}^{\prime} \Lambda_{0} \phi_{0}-\tilde{\phi}_{A}^{\prime}\left(\Lambda_{0}+Z_{A}^{\prime} Z_{A}\right) \tilde{\phi}_{A} .
\end{aligned}
$$

It follows from (A.26) that

$$
\begin{aligned}
\phi_{A} \mid R_{P}, r_{A}, \sigma_{u} & \sim N\left(\tilde{\phi}_{A}, \sigma_{u}^{2}\left(\Lambda_{0}+Z_{A}^{\prime} Z_{A}\right)^{-1}\right) \\
\sigma_{u}^{2} \mid R_{P}, r_{A} & \sim \frac{h_{A}}{\chi_{T+\nu_{0}}^{2}}
\end{aligned}
$$

and hence

$$
\begin{aligned}
\tilde{\sigma}_{u}^{2}=E\left(\sigma_{u}^{2} \mid R_{P}, r_{A}\right) & =\frac{h_{A}}{T+\nu_{0}-2} \\
\operatorname{Var}\left(\phi_{A} \mid R_{P}, r_{A}\right) & =\tilde{\sigma}_{u}^{2}\left(\Lambda_{0}+Z_{A}^{\prime} Z_{A}\right)^{-1}
\end{aligned}
$$

where the last equation follows from variance decomposition.

\section{A.3. Posterior moments of a fund's alpha}

Let $\tilde{\alpha}_{N}$ and $V_{\alpha_{N}}$ denote the posterior mean and covariance matrix of $\alpha_{N}$, given by the appropriate submatrices of the moments in (A.13) and (A.15), and let $V_{\phi_{A}}$ denote the posterior covariance matrix of $\phi_{A}$ given in (A.32). From the previous discussion recall that the posteriors of $\phi_{A}$ and $\alpha_{N}$ are independent. Thus, from equation (7), the posterior mean of the fund's alpha is given by

$$
\tilde{\alpha}_{A}=\tilde{\delta}_{A}+\tilde{c}_{A N}^{\prime} \tilde{\alpha}_{N}
$$

where $\tilde{\delta}_{A}$ and $\tilde{c}_{A N}$ are subvectors of the posterior mean of $\phi_{A}$ given in (A.27).

To obtain the posterior variance of $\alpha_{A}$, rewrite (7) as

$$
\alpha_{A}=\phi_{A}^{\prime} d
$$

where $d=\left(\begin{array}{lll}1 & \alpha_{N}^{\prime} & 0\end{array}\right)^{\prime}$, and the posterior mean and covariance matrix of $d$ are given by

$$
\tilde{d}=\left[\begin{array}{c}
1 \\
\tilde{\alpha}_{N} \\
0
\end{array}\right], \quad V_{d}=\left[\begin{array}{ccc}
0 & 0 & 0 \\
0 & V_{\alpha_{N}} & 0 \\
0 & 0 & 0
\end{array}\right]
$$

Let $\mathcal{D}$ denote the data, $R_{P}$ and $r_{A}$, and note that by the variance-decomposition rule,

$$
\operatorname{Var}\left(\alpha_{A} \mid \mathcal{D}\right)=\mathrm{E}\left[\operatorname{Var}\left(\phi_{A}^{\prime} d \mid \mathcal{D}, d\right) \mid \mathcal{D}\right]+\operatorname{Var}\left[\mathrm{E}\left(\phi_{A}^{\prime} d \mid \mathcal{D}, d\right) \mid \mathcal{D}\right]
$$


Using the independence of $\phi_{A}$ and $d$, the first term in (A.36) can expressed as

$$
\begin{aligned}
\mathrm{E}\left[\operatorname{Var}\left(\phi_{A}^{\prime} d \mid \mathcal{D}, d\right) \mid \mathcal{D}\right] & =\mathrm{E}\left[d^{\prime} V_{\phi_{A}} d \mid \mathcal{D}\right] \\
& =\mathrm{E}\left[\operatorname{tr}\left(V_{\phi_{A}} d d^{\prime}\right) \mid \mathcal{D}\right] \\
& =\operatorname{tr}\left[V_{\phi_{A}} \mathrm{E}\left(d d^{\prime} \mid \mathcal{D}\right)\right] \\
& =\operatorname{tr}\left(V_{\phi_{A}} V_{d}\right)+\tilde{d}^{\prime} V_{\phi_{A}} \tilde{d}
\end{aligned}
$$

and the second term can be expressed as

$$
\begin{aligned}
\operatorname{Var}\left[\mathrm{E}\left(\phi_{A}^{\prime} d \mid \mathcal{D}, d\right) \mid \mathcal{D}\right] & =\operatorname{Var}\left[\tilde{\phi}_{A} d \mid \mathcal{D}\right] \\
& =\tilde{\phi}_{A}^{\prime} V_{d} \tilde{\phi}_{A} .
\end{aligned}
$$

\section{A.4. Posterior distribution of a fund's Sharpe ratio}

A fund's Sharpe ratio, $S_{A}$, is defined as

$$
S_{A}=\frac{E_{A}}{\sigma_{A}} .
$$

The fund's expected return, $E_{A}$, is calculated from equation (5) as

$$
E_{A}=\delta_{A}+c_{A}^{\prime} E_{P}=\phi_{A}^{\prime}\left[\begin{array}{c}
1 \\
E_{P}
\end{array}\right],
$$

where

$$
E_{P}=\left[\begin{array}{c}
\alpha_{N}+B_{N} E_{B} \\
E_{B}
\end{array}\right]
$$

is the vector of expected returns on all passive assets. The fund's standard deviation of returns, $\sigma_{A}$, is calculated from the same equation as

$$
\sigma_{A}^{2}=c_{A}^{\prime} V_{P} c_{A}+\sigma_{u}^{2},
$$

where

$$
V_{P}=\left[\begin{array}{cc}
B_{N} V_{B B} B_{N}^{\prime}+\Sigma & B_{N} V_{B B} \\
V_{B B} B_{N}^{\prime} & V_{B B}
\end{array}\right]
$$

is the covariance matrix of returns on all passive assets. Independent posterior draws of $E_{A}$ and $\sigma_{A}$ are constructed by drawing parameters from their posterior distributions in equations (A.11), (A.12), (A.16), (A.17), (A.29), and (A.30). Using equation (A.39), these draws are then used to construct a large number of independent draws of $S_{A}$ from its posterior distribution. 


\section{A.5. Fund Classification by Objectives}

We select domestic equity funds from the 1998 CRSP Survivor Bias Free Mutual Fund Database, based on the information that the database provides about fund holdings, fund objectives, and fund names. In addition to international funds, money market funds, and bond funds, we exclude balanced funds, flexible funds, and funds of funds. We also exclude multiple share classes for the same fund, funds with at most 12 months of (continuous) return data, funds with unknown objectives, funds with no expense, turnover, or load data, and a small number of funds with apparent data errors. ${ }^{13}$

The resulting 2,609 domestic equity funds are assigned to one of seven broad investment objectives, using the information that the CRSP database provides about classifications by Wiesenberger ("OBJ"), ICDI ("ICDI_OBJ"), and Strategic Insight ("SI_OBJ"). Our classification is as follows: ${ }^{14}$

1. Small company growth - OBJ: SCG; SI_OBJ: SCG

2. Other aggressive growth - OBJ: AGG; ICDI_OBJ: AG, AGG; SI_OBJ: AGG

3. Growth - OBJ: G, G-S, S-G, GRO, LTG; ICDI_OBJ: LG; SI_OBJ: GRO

4. Income - OBJ: I, I-S, IEQ, ING; ICDI_OBJ: IN; SI_OBJ: ING

5. Growth and income - OBJ: GCI, G-I, G-I-S, G-S-I, I-G, I-G-S, I-S-G, S-G-I, S-I-G, GRI; ICDI_OBJ: GI; SI_OBJ: GRI

6. Maximum capital gains - OBJ: MCG

7. Sector funds - OBJ: ENR, FIN, HLT, TCH, UTL; ICDI_OBJ: SF, UT; SI_OBJ: ENV, FIN, HLT, TEC, UTI, RLE, NTR, SEC

A fund is included in a particular category in any given year if at least one of the objective classifications OBJ, ICDI_OBJ, SI_OBJ satisfies the above criterion. Funds are assigned to the categories in ascending order, beginning with category one. For example, if a fund is

\footnotetext{
${ }^{13}$ We made various corrections to the CRSP mutual fund data and also incorporated a number of corrections generously provided by Thomas Knox and the authors of Baks, Metrick, and Wachter (2001).

${ }^{14}$ Note that the Wiesenberger objective IEQ stands for "Income-Equity", not "International Equity" as stated in the 1998 CRSP manual. Also, the Wiesenberger objective LTG stands for "Long-term growth", not "Long-term government". The Strategic Insight codes "GMC" and "FOF", not mentioned in the manual, apparently denote "Growth: Mid-Cap" and "Fund of Funds", respectively. These corrections are easily confirmed by browsing the fund names.
} 
classified as small-company growth by Wiesenberger and as aggressive growth by ICDI, it is counted as small company growth, since that category comes before the aggressive growth category. If a fund's objective is not the same in each year, the fund is assigned to the category into which it is classified most often. 
Table 1

\section{Number of Equity Mutual Funds Classified by} History Length and Investment Objective

The sample contains domestic equity mutual funds in the CRSP database with continuous return histories longer than one year. Multiple share classes for the same fund are excluded. Funds are assigned to one of seven broad investment objectives using information that the CRSP database provides about classifications by Wiesenberger, ICDI, and Strategic Insight.

\begin{tabular}{lccccccc}
\hline & \multicolumn{7}{c}{ Length of fund's return history (months) } \\
\cline { 2 - 8 } Investment objective & $13-23$ & $24-35$ & $36-59$ & $60-119$ & $120-239$ & $\geq 240$ & All \\
\hline Small-company growth & 128 & 60 & 95 & 109 & 21 & 0 & 413 \\
Other aggressive growth & 40 & 30 & 41 & 32 & 10 & 0 & 153 \\
Growth & 213 & 130 & 226 & 251 & 92 & 60 & 972 \\
Income & 36 & 35 & 38 & 47 & 14 & 4 & 174 \\
Growth and income & 154 & 80 & 119 & 153 & 36 & 34 & 576 \\
Maximum capital gains & 9 & 10 & 17 & 16 & 13 & 41 & 106 \\
Sector funds & 61 & 37 & 45 & 68 & 4 & 0 & 215 \\
All funds & 641 & 382 & 581 & 676 & 190 & 139 & 2609 \\
\hline
\end{tabular}


Table 2

Estimates of Alpha for Equity Mutual Funds

Each value in the table is the median across the set of designated funds, expressed in percent per annum. Fund performance, denoted by $\alpha_{A}$, is defined as the intercept in the regression of the fund's excess return, $r_{A, t}$, on either the market benchmark index, $\mathrm{MKT}_{t},($ Panel A) or that benchmark index plus the size and value benchmark indexes, $\mathrm{SMB}_{t}$ and $\mathrm{HML}_{t}$ (Panel B). The OLS estimate of $\alpha_{A}$, denoted by $\hat{\alpha}_{A}$, is based on the fund's available history and the corresponding history of the benchmarks. The posterior mean of $\alpha_{A}$, denoted by $\tilde{\alpha}_{A}$, is based on the fund's available history as well as the returns from July 1963 through December 1998 on the benchmarks and additional non-benchmark indexes. The quantity $\sigma_{\alpha_{N}}$, expressed in percent per annum, denotes the prior standard deviation of the intercept $\alpha_{N}$ in a regression of a nonbenchmark return on the benchmark indexes. The prior for $\alpha_{A}$ is diffuse.

\begin{tabular}{|c|c|c|c|c|c|c|c|}
\hline \multirow{2}{*}{$\begin{array}{l}\text { History length or } \\
\text { investment objective }\end{array}$} & \multirow[b]{2}{*}{$\hat{\alpha}_{A}$} & \multicolumn{3}{|c|}{$\tilde{\alpha}_{A}$ for $\sigma_{\alpha_{N}}$ of } & \multicolumn{3}{|c|}{$\left|\hat{\alpha}_{A}-\tilde{\alpha}_{A}\right|$ for $\sigma_{\alpha_{N}}$ of } \\
\hline & & 0 & $2 \%$ & $\infty$ & 0 & $2 \%$ & $\infty$ \\
\hline \multicolumn{8}{|c|}{ A. $r_{A, t}=\alpha_{A}+\beta_{A} \mathrm{MKT}_{t}+\epsilon_{A, t}$} \\
\hline 13-23 months & -4.81 & -2.07 & -1.87 & -1.34 & 3.27 & 3.29 & 3.33 \\
\hline 24-35 months & -2.85 & -1.64 & -1.53 & -1.17 & 2.53 & 2.47 & 2.72 \\
\hline 36-59 months & -2.87 & -1.61 & -1.35 & -1.13 & 2.44 & 2.24 & 2.10 \\
\hline 60-119 months & -1.49 & -0.97 & -0.91 & -0.56 & 1.35 & 1.29 & 1.42 \\
\hline 120-239 months & -0.84 & -0.09 & -0.08 & 0.03 & 1.29 & 1.04 & 0.96 \\
\hline 240 months and greater & -0.53 & -0.17 & -0.26 & -0.14 & 0.70 & 0.53 & 0.17 \\
\hline Small-company growth & -8.45 & -1.59 & -0.97 & -0.05 & 7.20 & 7.66 & 8.30 \\
\hline Other aggressive growth & -5.41 & -0.97 & -0.74 & -1.06 & 4.80 & 4.65 & 4.58 \\
\hline Growth & -2.17 & -0.97 & -1.01 & -1.17 & 1.64 & 1.48 & 1.52 \\
\hline Income & -0.39 & -1.84 & -1.40 & -0.45 & 1.27 & 1.07 & 0.83 \\
\hline Growth and income & -0.51 & -0.97 & -0.87 & -0.59 & 0.93 & 0.89 & 1.02 \\
\hline Maximum capital gains & -2.29 & -1.47 & -1.53 & -1.95 & 2.16 & 1.75 & 1.34 \\
\hline Sector funds & -1.06 & -3.96 & -2.70 & 0.09 & 4.95 & 3.48 & 2.95 \\
\hline All funds & -2.13 & -1.25 & -1.07 & -0.74 & 2.05 & 1.87 & 1.90 \\
\hline \multicolumn{8}{|c|}{ B. $r_{A, t}=\alpha_{A}+b_{A, 1} \mathrm{MKT}_{t}+b_{A, 2} \mathrm{SMB}_{t}+b_{A, 3} \mathrm{HML}_{t}+\eta_{A, t}$} \\
\hline $13-23$ months & -1.68 & -2.07 & -1.96 & -1.43 & 1.66 & 1.55 & 1.59 \\
\hline 24-35 months & -1.63 & -1.64 & -1.52 & -1.38 & 1.40 & 1.25 & 1.01 \\
\hline 36-59 months & -1.29 & -1.61 & -1.46 & -1.14 & 1.05 & 0.95 & 0.78 \\
\hline 60-119 months & -0.92 & -0.97 & -0.94 & -0.66 & 0.76 & 0.57 & 0.39 \\
\hline 120-239 months & 0.07 & -0.09 & -0.06 & 0.08 & 0.64 & 0.42 & 0.24 \\
\hline 240 months and greater & 0.12 & -0.17 & -0.13 & 0.17 & 0.76 & 0.50 & 0.05 \\
\hline Small-company growth & -0.41 & -1.59 & -1.16 & -0.08 & 1.45 & 1.15 & 0.92 \\
\hline Other aggressive growth & -0.37 & -0.97 & -0.45 & 0.08 & 1.76 & 1.34 & 0.96 \\
\hline Growth & -0.88 & -0.97 & -0.86 & -0.72 & 0.90 & 0.78 & 0.59 \\
\hline Income & -2.03 & -1.84 & -1.90 & -1.74 & 0.74 & 0.61 & 0.47 \\
\hline Growth and income & -1.19 & -0.97 & -1.00 & -1.11 & 0.79 & 0.68 & 0.44 \\
\hline Maximum capital gains & -0.28 & -1.47 & -1.32 & -0.34 & 1.40 & 1.03 & 0.45 \\
\hline Sector funds & -1.84 & -3.96 & -3.51 & -2.48 & 3.18 & 2.44 & 1.35 \\
\hline All funds & -1.07 & -1.25 & -1.14 & -0.86 & 1.09 & 0.91 & 0.65 \\
\hline
\end{tabular}


Table 3

Average Equity-Fund Alphas: Are They Negative?

The table reports the posterior mean of $\bar{\alpha}_{A}$, the average $\alpha_{A}$ across the set of designated funds, expressed in percent per annum. Also reported is the posterior probability (expressed in percent) that $\bar{\alpha}_{A}$ is negative. Fund performance, denoted by $\alpha_{A}$, is defined as the intercept in the regression of the fund's excess return, $r_{A, t}$, on either the market benchmark index, $\mathrm{MKT}_{t},($ Panel A) or that benchmark index plus the size and value benchmark indexes, $\mathrm{SMB}_{t}$ and $\mathrm{HML}_{t}$ (Panel B). The OLS estimate of $\alpha_{A}$, denoted by $\hat{\alpha}_{A}$, is based on the fund's available history and the corresponding history of the benchmarks. The posterior mean of $\alpha_{A}$, denoted by $\tilde{\alpha}_{A}$, is based on the fund's available history as well as the returns from July 1963 through December 1998 on the benchmarks and additional non-benchmark indexes. The quantity $\sigma_{\alpha_{N}}$, expressed in percent per annum, denotes the prior standard deviation of the intercept $\alpha_{N}$ in a regression of a nonbenchmark return on the benchmark indexes. The prior for $\alpha_{A}$ is diffuse.

\begin{tabular}{|c|c|c|c|c|c|c|}
\hline \multirow{3}{*}{$\begin{array}{l}\text { History length or } \\
\text { investment objective }\end{array}$} & \multicolumn{3}{|c|}{ Posterior mean of $\bar{\alpha}_{A}$} & \multicolumn{3}{|c|}{$\operatorname{Prob}\left(\bar{\alpha}_{A}<0\right)$} \\
\hline & \multirow[b]{2}{*}{$\hat{\alpha}_{A}$} & \multicolumn{2}{|c|}{$\tilde{\alpha}_{A}$ for $\sigma_{\alpha_{N}}$ of } & \multirow[b]{2}{*}{$\hat{\alpha}_{A}$} & \multicolumn{2}{|c|}{$\tilde{\alpha}_{A}$ for $\sigma_{\alpha_{N}}$ of } \\
\hline & & 0 & $\infty$ & & 0 & $\infty$ \\
\hline \multicolumn{7}{|c|}{ A. $r_{A, t}=\alpha_{A}+\beta_{A} \mathrm{MKT}_{t}+\epsilon_{A, t}$} \\
\hline $13-23$ months & -6.31 & -1.67 & -1.19 & 100 & 100 & 98 \\
\hline 24-35 months & -4.77 & -1.67 & -1.29 & 100 & 100 & 98 \\
\hline 36-59 months & -4.12 & -1.78 & -1.39 & 100 & 100 & 100 \\
\hline 60-119 months & -2.13 & -0.99 & -0.66 & 100 & 100 & 94 \\
\hline 120-239 months & -1.07 & -0.08 & 0.11 & 100 & 72 & 42 \\
\hline 240 months and greater & -0.50 & -0.27 & -0.26 & 100 & 99 & 70 \\
\hline Small-company growth & -9.29 & -1.28 & 0.01 & 100 & 100 & 50 \\
\hline Other aggressive growth & -6.79 & -0.99 & -1.39 & 100 & 98 & 89 \\
\hline Growth & -3.06 & -1.00 & -1.27 & 100 & 100 & 100 \\
\hline Income & -0.84 & -1.56 & -0.36 & 100 & 100 & 80 \\
\hline Growth and income & -1.02 & -1.25 & -1.01 & 100 & 100 & 100 \\
\hline Maximum capital gains & -4.74 & -2.85 & -3.01 & 100 & 100 & 100 \\
\hline Sector funds & -4.14 & -2.45 & -0.54 & 100 & 100 & 78 \\
\hline All funds & -3.83 & -1.33 & -0.97 & 100 & 100 & 98 \\
\hline \multicolumn{7}{|c|}{ B. $r_{A, t}=\alpha_{A}+b_{A, 1} \mathrm{MKT}_{t}+b_{A, 2} \mathrm{SMB}_{t}+b_{A, 3} \mathrm{HML}_{t}+\eta_{A, t}$} \\
\hline $13-23$ months & -1.54 & -1.67 & -1.06 & 100 & 100 & 100 \\
\hline 24-35 months & -1.22 & -1.67 & -1.02 & 100 & 100 & 100 \\
\hline 36-59 months & -1.32 & -1.78 & -1.10 & 100 & 100 & 100 \\
\hline 60-119 months & -0.66 & -0.99 & -0.44 & 100 & 100 & 99 \\
\hline 120-239 months & 0.38 & -0.08 & 0.46 & 0 & 72 & 1 \\
\hline 240 months and greater & 0.15 & -0.27 & 0.21 & 10 & 99 & 17 \\
\hline Small-company growth & -0.00 & -1.28 & 0.50 & 50 & 100 & 9 \\
\hline Other aggressive growth & 0.27 & -0.99 & 0.29 & 30 & 98 & 30 \\
\hline Growth & -0.78 & -1.00 & -0.61 & 100 & 100 & 99 \\
\hline Income & -2.04 & -1.56 & -1.64 & 100 & 100 & 100 \\
\hline Growth and income & -1.76 & -1.25 & -1.43 & 100 & 100 & 100 \\
\hline Maximum capital gains & -1.67 & -2.85 & -1.64 & 100 & 100 & 100 \\
\hline Sector funds & -1.43 & -2.45 & -1.25 & 99 & 100 & 99 \\
\hline All funds & -0.99 & -1.33 & -0.72 & 100 & 100 & 100 \\
\hline
\end{tabular}


Table 4

\section{Relative Precision of Estimates of Alpha for Equity Mutual Funds}

Fund performance, denoted by $\alpha_{A}$, is defined as the intercept in the regression of the fund's excess return, $r_{A, t}$, on either the market benchmark index, $\mathrm{MKT}_{t}$, (Panel A) or that benchmark index plus the size and value benchmark indexes, $\mathrm{SMB}_{t}$ and $\mathrm{HML}_{t}$ (Panel B). The OLS estimate of $\alpha_{A}$, denoted by $\hat{\alpha}_{A}$, is based on the fund's available history and the corresponding history of the benchmarks. The posterior mean of $\alpha_{A}$, denoted by $\tilde{\alpha}_{A}$, is based on the fund's available history as well as the returns from July 1963 through December 1998 on the benchmarks and additional non-benchmark indexes. The quantity $\sigma_{\alpha_{N}}$, expressed in percent per annum, denotes the prior standard deviation of the intercept $\alpha_{N}$ in a regression of a nonbenchmark return on the benchmark indexes. The prior for $\alpha_{A}$ is diffuse.

\begin{tabular}{|c|c|c|c|c|c|c|}
\hline \multirow{2}{*}{$\begin{array}{l}\text { History length or } \\
\text { investment objective }\end{array}$} & \multicolumn{2}{|c|}{$\sigma_{\alpha_{N}}=0$} & \multicolumn{2}{|c|}{$\sigma_{\alpha_{N}}=2 \%$} & \multicolumn{2}{|c|}{$\sigma_{\alpha_{N}}=\infty$} \\
\hline & median & $\%<1$ & median & $\%<1$ & median & $\%<1$ \\
\hline \multicolumn{7}{|c|}{ A. $r_{A, t}=\alpha_{A}+\beta_{A} \mathrm{MKT}_{t}+\epsilon_{A, t}$} \\
\hline 13-23 months & 0.62 & 83 & 0.62 & 83 & 0.63 & 82 \\
\hline 24-35 months & 0.63 & 88 & 0.62 & 88 & 0.63 & 89 \\
\hline 36-59 months & 0.65 & 90 & 0.65 & 92 & 0.68 & 91 \\
\hline 60-119 months & 0.70 & 93 & 0.69 & 97 & 0.73 & 96 \\
\hline 120-239 months & 0.76 & 93 & 0.77 & 97 & 0.84 & 98 \\
\hline 240 months and greater & 0.84 & 88 & 0.87 & 92 & 0.98 & 74 \\
\hline Small-company growth & 0.33 & 100 & 0.34 & 100 & 0.39 & 100 \\
\hline Other aggressive growth & 0.54 & 90 & 0.55 & 90 & 0.59 & 90 \\
\hline Growth & 0.76 & 87 & 0.76 & 89 & 0.80 & 87 \\
\hline Income & 0.75 & 84 & 0.75 & 89 & 0.77 & 89 \\
\hline Growth and income & 0.77 & 83 & 0.77 & 85 & 0.80 & 85 \\
\hline Maximum capital gains & 0.70 & 92 & 0.73 & 95 & 0.85 & 88 \\
\hline Sector funds & 0.62 & 96 & 0.63 & 96 & 0.66 & 95 \\
\hline All funds & 0.68 & 89 & 0.68 & 91 & 0.72 & 89 \\
\hline \multicolumn{7}{|c|}{ B. $r_{A, t}=\alpha_{A}+b_{A, 1} \mathrm{MKT}_{t}+b_{A, 2} \mathrm{SMB}_{t}+b_{A, 3} \mathrm{HML}_{t}+\eta_{A, t}$} \\
\hline $13-23$ months & 0.70 & 81 & 0.69 & 81 & 0.69 & 81 \\
\hline 24-35 months & 0.79 & 76 & 0.78 & 79 & 0.78 & 77 \\
\hline 36-59 months & 0.85 & 80 & 0.84 & 83 & 0.83 & 82 \\
\hline 60-119 months & 0.89 & 80 & 0.88 & 84 & 0.89 & 83 \\
\hline 120-239 months & 0.95 & 75 & 0.93 & 88 & 0.94 & 91 \\
\hline 240 months and greater & 1.00 & 53 & 0.98 & 71 & 0.99 & 83 \\
\hline Small-company growth & 0.80 & 84 & 0.79 & 86 & 0.80 & 86 \\
\hline Other aggressive growth & 0.81 & 86 & 0.79 & 88 & 0.78 & 88 \\
\hline Growth & 0.88 & 78 & 0.86 & 82 & 0.88 & 82 \\
\hline Income & 0.88 & 79 & 0.87 & 83 & 0.86 & 82 \\
\hline Growth and income & 0.91 & 67 & 0.89 & 72 & 0.90 & 73 \\
\hline Maximum capital gains & 0.96 & 66 & 0.94 & 75 & 0.96 & 83 \\
\hline Sector funds & 0.74 & 95 & 0.74 & 95 & 0.76 & 95 \\
\hline All funds & 0.86 & 78 & 0.84 & 82 & 0.85 & 82 \\
\hline
\end{tabular}




\section{Table 5}

\section{Comparison of Estimated CAPM and Fama-French Alphas under Alternative Roles for Non-Benchmark Assets}

Each entry in Panels A and B is the median across funds of the absolute difference between posterior means of a fund's CAPM alpha and its Fama-French alpha, in percent per annum, under alternative prior beliefs about the degree to which each model prices the non-benchmark assets. Each entry in Panel C is the median absolute difference of the OLS alpha estimates. Fund performance, denoted by $\alpha_{A}$, is defined as the intercept in the regression of the fund's excess return, $r_{A, t}$, on either the market benchmark index, $\mathrm{MKT}_{t},(\mathrm{CAPM})$ or that benchmark index plus the size and value benchmark indexes, $\mathrm{SMB}_{t}$ and $\mathrm{HML}_{t}$ (Fama-French). The OLS estimate of $\alpha_{A}$, denoted by $\hat{\alpha}_{A}$, is based on the fund's available history and the corresponding history of the benchmarks. The posterior mean of $\alpha_{A}$, denoted by $\tilde{\alpha}_{A}$, is based on the fund's available history as well as the returns from July 1963 through December 1998 on the benchmark and additional non-benchmark indexes. The quantity $\sigma_{\alpha_{N}}$ denotes the prior standard deviation of the intercept $\alpha_{N}$ in a regression of a non-benchmark return on the benchmark returns, and the table compares $\tilde{\alpha}_{A}$ under a given non-zero $\sigma_{\alpha_{N}}$ but different specifications of the set of benchmark indexes. (The posterior means of the CAPM and Fama-French alphas are identical under $\sigma_{\alpha_{N}}=0$.) The prior for $\alpha_{A}$ is diffuse.

\begin{tabular}{|c|c|c|c|c|c|c|c|}
\hline \multirow[b]{2}{*}{ Investment objective } & \multicolumn{7}{|c|}{ Length of fund's return history (months) } \\
\hline & $13-23$ & $24-35$ & $36-59$ & $60-119$ & $120-239$ & $\geq 240$ & All \\
\hline \multicolumn{8}{|c|}{ A. Mispricing uncertainty for the non-benchmark assets $\left(\sigma_{\alpha_{N}}\right)$ equal to $2 \%$ per annum } \\
\hline Small-company growth & 0.58 & 1.01 & 0.74 & 0.67 & 0.70 & na & 0.69 \\
\hline Other aggressive growth & 0.69 & 0.43 & 0.74 & 0.60 & 0.69 & na & 0.67 \\
\hline Growth & 0.43 & 0.38 & 0.41 & 0.41 & 0.40 & 0.35 & 0.40 \\
\hline Income & 0.48 & 0.38 & 0.47 & 0.49 & 0.57 & 0.41 & 0.47 \\
\hline Growth and income & 0.18 & 0.20 & 0.26 & 0.19 & 0.33 & 0.23 & 0.20 \\
\hline Maximum capital gains & 0.64 & 0.35 & 0.43 & 0.66 & 0.46 & 0.37 & 0.45 \\
\hline Sector funds & 0.96 & 0.85 & 1.36 & 0.93 & 1.11 & na & 0.98 \\
\hline All funds & 0.42 & 0.41 & 0.47 & 0.43 & 0.40 & 0.32 & 0.42 \\
\hline \multicolumn{8}{|c|}{ B. No reliance on the model to price the non-benchmark assets $\left(\sigma_{\alpha_{N}}=\infty\right)$} \\
\hline Small-company growth & 1.69 & 2.72 & 2.11 & 1.89 & 2.02 & na & 2.03 \\
\hline Other aggressive growth & 2.10 & 1.29 & 2.38 & 1.75 & 2.20 & na & 1.89 \\
\hline Growth & 1.25 & 1.11 & 1.18 & 1.13 & 1.13 & 1.02 & 1.14 \\
\hline Income & 1.31 & 1.07 & 1.29 & 1.27 & 1.52 & 1.17 & 1.27 \\
\hline Growth and income & 0.53 & 0.63 & 0.74 & 0.54 & 0.89 & 0.61 & 0.59 \\
\hline Maximum capital gains & 2.00 & 1.16 & 1.64 & 2.15 & 1.30 & 1.12 & 1.34 \\
\hline Sector funds & 2.43 & 2.41 & 3.40 & 2.37 & 2.91 & na & 2.47 \\
\hline All funds & 1.18 & 1.18 & 1.38 & 1.26 & 1.20 & 0.99 & 1.24 \\
\hline \multicolumn{8}{|c|}{ C. No use of non-benchmark assets (OLS estimates) } \\
\hline Small-company growth & 11.21 & 11.33 & 7.98 & 4.72 & 3.96 & na & 8.07 \\
\hline Other aggressive growth & 8.97 & 6.79 & 6.43 & 4.47 & 3.52 & na & 6.35 \\
\hline Growth & 3.60 & 1.97 & 1.91 & 1.28 & 1.33 & 1.13 & 1.81 \\
\hline Income & 1.80 & 1.43 & 1.30 & 1.44 & 1.62 & 0.93 & 1.44 \\
\hline Growth and income & 1.79 & 1.73 & 1.54 & 1.01 & 0.85 & 0.68 & 1.25 \\
\hline Maximum capital gains & 5.45 & 5.67 & 3.04 & 3.61 & 2.30 & 1.46 & 2.60 \\
\hline Sector funds & 5.08 & 5.08 & 1.51 & 2.90 & 3.60 & na & 3.29 \\
\hline All funds & 3.88 & 2.93 & 2.47 & 1.67 & 1.53 & 1.07 & 2.28 \\
\hline
\end{tabular}




\section{Table 6}

\section{Comparison of Estimated Alphas With and Without the Pricing-Model Restriction Applied to the Non-benchmark Assets}

Each entry in the table is the median across funds of the absolute difference between posterior means of a fund's alpha, in percent per annum, under alternative prior beliefs about whether the pricing-model restriction applies to the non-benchmark assets. Fund performance, denoted by $\alpha_{A}$, is defined as the intercept in the regression of the fund's excess return, $r_{A, t}$, on either the market benchmark index, $\mathrm{MKT}_{t}$, $($ Panel A) or that benchmark index plus the size and value benchmark indexes, $\mathrm{SMB}_{t}$ and $\mathrm{HML}_{t}(\mathrm{Panel} \mathrm{B})$. The posterior mean of $\alpha_{A}$, denoted by $\tilde{\alpha}_{A}$, is based on the fund's available history as well as the returns from July 1963 through December 1998 on the benchmarks and additional non-benchmark indexes. The quantity $\sigma_{\alpha_{N}}$ denotes the prior standard deviation of the intercept $\alpha_{N}$ in a regression of a non-benchmark return on the benchmark indexes, and the table compares $\tilde{\alpha}_{A}$ under $\sigma_{\alpha_{N}}=0$ versus $\sigma_{\alpha_{N}}=\infty$. The prior for $\alpha_{A}$ is diffuse.

\begin{tabular}{|c|c|c|c|c|c|c|c|}
\hline \multirow[b]{2}{*}{ Investment objective } & \multicolumn{7}{|c|}{ Length of fund's return history (months) } \\
\hline & $13-23$ & $24-35$ & $36-59$ & $60-119$ & $120-239$ & $\geq 240$ & All \\
\hline \multicolumn{8}{|c|}{ A. $r_{A, t}=\alpha_{A}+\beta_{A} \mathrm{MKT}_{t}+\epsilon_{A, t}$} \\
\hline Small-company growth & 1.69 & 1.40 & 1.46 & 1.57 & 1.08 & na & 1.55 \\
\hline Other aggressive growth & 0.72 & 0.67 & 0.88 & 0.82 & 0.92 & na & 0.77 \\
\hline Growth & 0.78 & 0.75 & 0.85 & 0.84 & 0.91 & 0.83 & 0.83 \\
\hline Income & 1.32 & 1.00 & 1.30 & 1.23 & 1.56 & 1.19 & 1.28 \\
\hline Growth and income & 0.48 & 0.56 & 0.69 & 0.52 & 0.64 & 0.48 & 0.54 \\
\hline Maximum capital gains & 0.85 & 0.93 & 1.36 & 1.35 & 1.45 & 0.95 & 1.07 \\
\hline Sector funds & 3.31 & 2.94 & 6.22 & 3.61 & 4.78 & na & 4.31 \\
\hline All funds & 0.90 & 0.89 & 1.01 & 1.00 & 0.99 & 0.80 & 0.94 \\
\hline \multicolumn{8}{|c|}{ B. $r_{A, t}=\alpha_{A}+b_{A, 1} \mathrm{MKT}_{t}+b_{A, 2} \mathrm{SMB}_{t}+b_{A, 3} \mathrm{HML}_{t}+\eta_{A, t}$} \\
\hline Small-company growth & 1.58 & 2.05 & 1.39 & 1.62 & 1.76 & na & 1.62 \\
\hline Other aggressive growth & 1.88 & 1.20 & 1.90 & 1.78 & 1.19 & na & 1.63 \\
\hline Growth & 0.56 & 0.63 & 0.55 & 0.57 & 0.65 & 0.67 & 0.59 \\
\hline Income & 0.33 & 0.40 & 0.51 & 0.41 & 0.84 & 0.67 & 0.40 \\
\hline Growth and income & 0.34 & 0.37 & 0.41 & 0.36 & 0.46 & 0.36 & 0.37 \\
\hline Maximum capital gains & 1.49 & 1.33 & 1.91 & 1.41 & 0.89 & 1.30 & 1.29 \\
\hline Sector funds & 1.25 & 1.30 & 2.85 & 2.16 & 2.01 & na & 1.93 \\
\hline All funds & 0.66 & 0.66 & 0.68 & 0.66 & 0.73 & 0.74 & 0.68 \\
\hline
\end{tabular}




\section{Table 7 \\ Estimates of Sharpe Ratios for Equity Mutual Funds}

Each value in the table is the median across the set of designated funds. A fund's Sharpe ratio is denoted as $S_{A}$. The estimate $\hat{S}_{A}$ is based solely on the fund's available history. The posterior mean of $S_{A}$, denoted by $\tilde{S}_{A}$, is based on the fund's available history as well as the returns from July 1963 through December 1998 on benchmark and non-benchmark passive indexes. All results are reported in annualized terms. The results for the CAPM and the Fama-French model are reported for prior beliefs in which the pricing relations are assumed to hold exactly. For the "No Model" results, prior beliefs in any pricing model are diffuse.

\begin{tabular}{|c|c|c|c|c|c|c|c|}
\hline \multirow{2}{*}{$\begin{array}{l}\text { History length or } \\
\text { investment objective }\end{array}$} & \multirow[b]{2}{*}{$\hat{S}_{A}$} & \multicolumn{2}{|c|}{ CAPM } & \multicolumn{2}{|c|}{ Fama-French } & \multicolumn{2}{|c|}{ No Model } \\
\hline & & $\tilde{S}_{A}$ & $\left|\hat{S}_{A}-\tilde{S}_{A}\right|$ & $\tilde{S}_{A}$ & $\left|\hat{S}_{A}-\tilde{S}_{A}\right|$ & $\tilde{S}_{A}$ & $\left|\hat{S}_{A}-\tilde{S}_{A}\right|$ \\
\hline $13-23$ months & 0.59 & 0.26 & 0.39 & 0.29 & 0.37 & 0.30 & 0.37 \\
\hline 24-35 months & 0.72 & 0.28 & 0.46 & 0.30 & 0.45 & 0.31 & 0.43 \\
\hline 36-59 months & 0.82 & 0.29 & 0.56 & 0.31 & 0.52 & 0.32 & 0.52 \\
\hline 60-119 months & 0.79 & 0.33 & 0.47 & 0.35 & 0.43 & 0.35 & 0.43 \\
\hline 120-239 months & 0.61 & 0.38 & 0.23 & 0.39 & 0.20 & 0.39 & 0.21 \\
\hline 240 months and greater & 0.36 & 0.38 & 0.07 & 0.37 & 0.06 & 0.37 & 0.03 \\
\hline Small-company growth & 0.51 & 0.29 & 0.23 & 0.27 & 0.25 & 0.36 & 0.17 \\
\hline Other aggressive growth & 0.68 & 0.31 & 0.32 & 0.26 & 0.39 & 0.33 & 0.30 \\
\hline Growth & 0.71 & 0.34 & 0.41 & 0.33 & 0.43 & 0.32 & 0.42 \\
\hline Income & 0.87 & 0.26 & 0.61 & 0.41 & 0.48 & 0.36 & 0.53 \\
\hline Growth and income & 0.91 & 0.34 & 0.58 & 0.42 & 0.51 & 0.36 & 0.58 \\
\hline Maximum capital gains & 0.14 & 0.30 & 0.17 & 0.22 & 0.15 & 0.27 & 0.12 \\
\hline Sector funds & 0.65 & 0.08 & 0.49 & 0.23 & 0.37 & 0.31 & 0.32 \\
\hline All funds & 0.68 & 0.31 & 0.41 & 0.33 & 0.39 & 0.34 & 0.38 \\
\hline
\end{tabular}


Table 8

\section{Relative Precision of Estimates of Sharpe Ratios for Equity Mutual Funds}

The table reports statistics for the ratio of variances, $\operatorname{var}\left(\tilde{S}_{A}\right) / \operatorname{var}\left(\hat{S}_{A}\right)$. A fund's Sharpe ratio is denoted as $S_{A}$. The estimate $\hat{S}_{A}$ is based solely on the fund's available history. The posterior mean of $S_{A}$, denoted by $\tilde{S}_{A}$, is based on the fund's available history as well as the returns from July 1963 through December 1998 on benchmark and non-benchmark passive indexes. The results for the CAPM and the Fama-French model are reported for prior beliefs in which the pricing relations are assumed to hold exactly. For the "No Model" results, prior beliefs in any pricing model are diffuse.

\begin{tabular}{|c|c|c|c|c|c|c|}
\hline \multirow{2}{*}{$\begin{array}{l}\text { History length or } \\
\text { investment objective }\end{array}$} & \multicolumn{2}{|c|}{ CAPM } & \multicolumn{2}{|c|}{ Fama-French } & \multicolumn{2}{|c|}{ No Model } \\
\hline & median & $\%<1$ & median & $\%<1$ & median & $\%<1$ \\
\hline $13-23$ months & 0.16 & 100 & 0.18 & 100 & 0.16 & 100 \\
\hline 24-35 months & 0.18 & 100 & 0.19 & 100 & 0.18 & 100 \\
\hline 36-59 months & 0.20 & 100 & 0.22 & 100 & 0.21 & 100 \\
\hline 60-119 months & 0.25 & 100 & 0.27 & 100 & 0.26 & 100 \\
\hline 120-239 months & 0.42 & 100 & 0.44 & 100 & 0.44 & 100 \\
\hline 240 months and greater & 0.87 & 89 & 0.88 & 91 & 0.88 & 93 \\
\hline Small-company growth & 0.19 & 100 & 0.21 & 100 & 0.21 & 100 \\
\hline Other aggressive growth & 0.23 & 100 & 0.24 & 100 & 0.24 & 100 \\
\hline Growth & 0.23 & 100 & 0.25 & 100 & 0.23 & 100 \\
\hline Income & 0.22 & 100 & 0.24 & 100 & 0.22 & 100 \\
\hline Growth and income & 0.19 & 98 & 0.21 & 98 & 0.19 & 99 \\
\hline Maximum capital gains & 0.47 & 100 & 0.46 & 100 & 0.46 & 99 \\
\hline Sector funds & 0.33 & 100 & 0.37 & 100 & 0.35 & 100 \\
\hline All funds & 0.22 & 99 & 0.24 & 99 & 0.23 & 100 \\
\hline
\end{tabular}




\section{Table 9}

\section{Comparison of Fund Rankings by Alternative Estimates of Sharpe Ratios}

The table compares rankings for 1585 equity mutual funds with at least three years of return history. Each entry in the table is the total number of funds satisfying the given ranking classifications for both estimates. A fund's Sharpe ratio is denoted as $S_{A}$. The estimate $\hat{S}_{A}$ is based solely on the fund's available history. The posterior mean of $S_{A}$, denoted by $\tilde{S}_{A}$, is based on the fund's available history as well as the returns from July 1963 through December 1998 on benchmark and non-benchmark passive indexes. Prior beliefs in any pricing model are diffuse.

\begin{tabular}{lccccc}
\hline Rankings & \multicolumn{5}{c}{ Rankings using $\hat{S}_{A}$} \\
\cline { 2 - 6 } using $\tilde{S}_{A}$ & Top 10\% & Next 22.5\% & Next 35\% & Next 22.5\% & Bottom 10\% \\
\hline Top 10\% & 35 & 54 & 63 & 4 & 3 \\
Next 22.5\% & 75 & 93 & 142 & 44 & 2 \\
Next 35\% & 43 & 160 & 192 & 142 & 18 \\
Next $22.5 \%$ & 6 & 47 & 135 & 120 & 49 \\
Bottom 10\% & 0 & 2 & 23 & 47 & 86 \\
\hline
\end{tabular}




\section{References}

Anderson, T.W., 1984. An Introduction to Multivariate Statistical Analysis. Wiley, New York.

Baks, K.P., Metrick, A., Wachter, J., 2001. Should investors avoid all actively managed mutual funds? A study in Bayesian performance evaluation. Journal of Finance 56, $45-85$.

Carhart, M.M., 1997. On persistence in mutual fund performance. Journal of Finance 52, 57-82.

Daniel, K., Titman, S., 1997. Evidence on the characteristics of cross sectional variation in stock returns. Journal of Finance 52, 1-33.

Daniel, K., Grinblatt, M., Titman, S., Wermers, R., 1997. Measuring mutual fund performance with characteristic-based benchmarks. Journal of Finance 52, 1035-1058.

Davis, J.L., Fama, E.F., French, K.R., 2000. Characteristics, covariances, and average returns: 1929 to 1997. Journal of Finance 55, 389-406.

Elton, E.J., Gruber, M.J., Blake, C.R., 1996. The persistence of risk-adjusted mutual fund performance. Journal of Business 69, 133-157.

Fama, E.F., French, K.R., 1993. Common risk factors in the returns on stocks and bonds. Journal of Financial Economics 33, 3-56.

Fama, E.F., French, K.R., 1996. Multifactor explanations of asset pricing anomalies. Journal of Finance 51, 55-84.

Ferson, W.E., Schadt, R.W., 1996. Measuring fund strategy and performance in changing economic conditions. Journal of Finance 51, 425-461.

Grinblatt, M., Titman, S., 1994. A study of monthly mutual fund returns and performance evaluation techniques. Journal of Financial and Quantitative Analysis 29, 419-444.

Grinblatt, M., Titman, S., 1995. Performance evaluation. In R. Jarrow, V. Maksimovic, and W. Ziemba, eds.: Handbook in Operations Research and Management Science, Vol. 9: Finance. Elsevier Science.

Hansen, L.P., 1982. Large Sample Properties of Generalized Method of Moments Estimators. Econometrica 50, 1029-1054.

Jegadeesh, N., Titman, S., 1993. Returns to buying winners and selling losers: Implications for stock market efficiency. Journal of Finance 48, 65-91.

Jensen, M.C., 1969. Risk, the pricing of capital assets, and the evaluation of investment portfolios. Journal of Business 42, 167-247.

Lehmann, B.N., Modest, D.M., 1987. Mutual fund performance evaluation: A comparison of benchmarks and benchmark comparisons. Journal of Finance 42, 233-265. 
Lintner, J., 1965. The valuation of risk assets and the selection of risky investments in stock portfolios and capital budgets. Review of Economics and Statistics 47, 13-37.

MacKinlay, A.C., 1995. Multifactor models do not explain deviations from the CAPM. Journal of Financial Economics 38, 3-28.

Moskowitz, T.J., Grinblatt, M., 1999. Do industries explain momentum? Journal of Finance 54, 1249-1290.

Pástor, Ľ., 2000. Portfolio selection and asset pricing models. Journal of Finance 55, 179223.

Pástor, Ľ., Stambaugh, R.F., 1999. Costs of equity capital and model mispricing. Journal of Finance, 54, 67-121.

Pástor, Ľ., Stambaugh, R.F., 2000. Comparing asset pricing models: an investment perspective. Journal of Financial Economics 56, 335-381.

Pástor, Ľ., Stambaugh, R.F., 2001. Investing in equity mutual funds. Unpublished working paper. University of Chicago and University of Pennsylvania.

Roll, R., 1978. Ambiguity when performance is measured by the securities market line. Journal of Finance 33, 1051-1069.

Ross, S.A., 1976. The arbitrage theory of capital asset pricing. Journal of Economic Theory $13,341-360$.

Shanken, J., 1990. Intertemporal asset pricing: An empirical investigation. Journal of Econometrics 45, 99-120.

Sharpe, W.F., 1964. Capital asset prices: A theory of market equilibrium under conditions of risk. Journal of Finance 19, 425-442.

Sharpe, W.F., 1992. Asset allocation: Management style and performance measurement. Journal of Portfolio Management 18 (2), 7-19.

Sharpe, W.F., 1997. Morningstar performance measures. World Wide Web, http://www.stanford.edu/ wfsharpe/art/stars/stars0.htm.

Sharpe, W.F., 1998. Morningstar's risk-adjusted ratings. Financial Analysts Journal 54 (4), $21-33$.

Stambaugh, R.F., 1997. Analyzing investments whose histories differ in length. Journal of Financial Economics 45, 285-331.

Theil, H., 1971. Principles of Econometrics. Wiley, New York.

Wermers, R., 2000. Mutual fund performance: An empirical decomposition into stockpicking talent, style, transactions costs, and expenses. Journal of Finance 55, 1655-1695.

Zellner, A., 1962. An efficient method of estimating seemingly unrelated regressions and tests for aggregation bias. Journal of the American Statistical Association 57, 348-368.

Zellner, A., 1971. An Introduction to Bayesian Inference in Econometrics. Wiley, New York. 